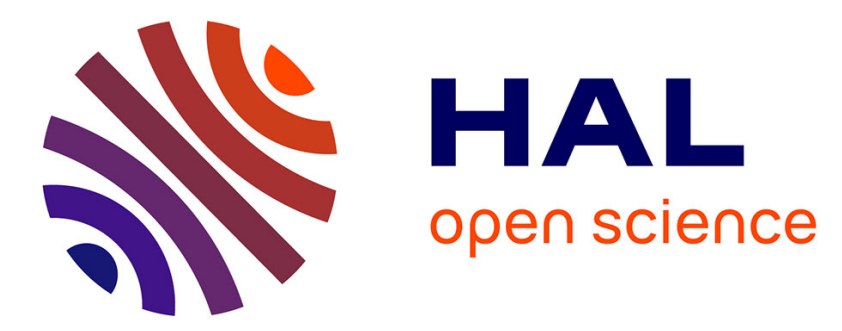

\title{
Computing the topology of a plane or space hyperelliptic curve
}

Juan Gerardo Alcázar, Jorge Caravantes, Gema M Diaz-Toca, Elias Tsigaridas

\section{To cite this version:}

Juan Gerardo Alcázar, Jorge Caravantes, Gema M Diaz-Toca, Elias Tsigaridas. Computing the topology of a plane or space hyperelliptic curve. Computer Aided Geometric Design, 2020, 10.1016/j.cagd.2020.101830 . hal-01968776v2

\section{HAL Id: hal-01968776 https://hal.inria.fr/hal-01968776v2}

Submitted on 27 Feb 2020

HAL is a multi-disciplinary open access archive for the deposit and dissemination of scientific research documents, whether they are published or not. The documents may come from teaching and research institutions in France or abroad, or from public or private research centers.
L'archive ouverte pluridisciplinaire HAL, est destinée au dépôt et à la diffusion de documents scientifiques de niveau recherche, publiés ou non, émanant des établissements d'enseignement et de recherche français ou étrangers, des laboratoires publics ou privés. 


\title{
Computing the topology of a plane or space hyperelliptic curve.
}

\author{
Juan Gerardo Alcázar ${ }^{\mathrm{a}, 1,2}$, Jorge Caravantes ${ }^{\mathrm{a}, 1}$, Gema M. Diaz-Toca ${ }^{\mathrm{b}, 1}$, \\ Elias Tsigaridas ${ }^{\mathrm{c}, 3}$ \\ ${ }^{a}$ Departamento de Física y Matemáticas, Universidad de Alcalá, E-28871 Madrid, Spain \\ ${ }^{b}$ Departamento de Ingeniería y Tecnología de Computadores, Universidad de Murcia, \\ E-30100 Murcia, Spain \\ ${ }^{c}$ Inria Paris and Institut de Mathématiques de Jussieu - Paris Rive Gauche, Sorbonne \\ Université and Paris Université, France
}

\begin{abstract}
We present algorithms to compute the topology of 2D and 3D hyperelliptic curves. The algorithms are based on the fact that $2 \mathrm{D}$ and $3 \mathrm{D}$ hyperelliptic curves can be seen as the image of a planar curve (the Weierstrass form of the curve), whose topology is easy to compute, under a birational mapping of the plane or the space. We report on a Maple implementation of these algorithms, and present several examples. Complexity and certification issues are also discussed.
\end{abstract}

\section{Introduction}

Rational curves are widely used in Computer Aided Geometric Design.

Hyperelliptic curves are not rational, but they are birationally equivalent to

Email addresses: juange.alcazar@uah.es (Juan Gerardo Alcázar),

jorge.caravantes@uah.e (Jorge Caravantes), gemadiaz@um.es ( Gema M. Diaz-Toca), elias.tsigaridas@inria.fr (Elias Tsigaridas)

${ }^{1}$ Supported by the Spanish Ministerio de Ciencia, Innovación y Universidades and by the European Regional Development Fund (ERDF), under the project MTM2017-88796-P.

${ }^{2}$ Member of the Research Group ASYNACS (Ref. CCEE2011/R34)

${ }^{3}$ Partially supported by a Giner de los Ríos Grant of the Universidad de Alcalá, ANR JCJC GALOP (ANR-17-CE40-0009), a public grant as part of the Investissement d'avenir project reference ANR-11-LABX-0056-LMH LabEx LMH (PGMO ALMA), the PHC GRAPE, and from the 2232 International Fellowship for Outstanding Researchers Program from TÜBITAK (Project No: 118C240). 
planar algebraic curves quadratic in one variable, the corresponding Weierstrass forms, where birationally equivalent means that there exists a rational mapping between the curve and its Weierstrass form with an also rational inverse. Since Weierstrass forms are quadratic in one variable, hyperelliptic curves are parametrizable by square-roots. Thus, hyperelliptic curves are one of the simplest examples of non-rational families of curves. Furthermore, this type of curves appears frequently in Computer Aided Geometric Design. A good account of the occurrence of hyperelliptic curves in this field is given in [8], where the problem of approximating hyperelliptic curves by means of rational parametrizations is addressed. As a brief summary of [8], non-rational offsets of rational planar curves and some bisector curves (line/rational curve, or circle/rational curve) are planar hyperelliptic curves. Contour curves of canal surfaces, intersections of two quadrics or intersections of a quadric and a ruled surface are examples of hyperelliptic curves in 3-space. With more generality, every planar or space algebraic curve $\mathcal{C}$ admitting a square-root parametrization (see also [27]) is hyperelliptic.

In this paper we address the problem of computing the topology of a hyperelliptic curve $\mathcal{C}$. Efficient and fast algorithms to compute the Weierstrass form $\mathcal{G}$ of $\mathcal{C}$, as well as a birational mapping $\mathbf{x}: \mathcal{G} \rightarrow \mathcal{C}$ can be found in many computer algebra systems, e.g. Sage, Maple or Magma. Here we will assume that $\mathbf{x}, \mathcal{G}$ are already known, and in fact that $\mathcal{C}$ is defined by means of the pair $\mathbf{x}, \mathcal{G}$, so that $\mathcal{C}$ is seen as the image of the planar algebraic curve $\mathcal{G}$ under the mapping defined by $\mathbf{x}$. Since $\mathcal{G}$ is a simple curve, quadratic in one variable, and therefore the union of the graphs of two univariate functions, the topology of $\mathcal{G}$ is very easy to capture. Thus, our strategy to compute the topology of $\mathcal{C}$ is to study how the birational mapping modifies the topology of the Weierstrass form. Hence, we might say that the Weierstrass form "guides" us to build the topology of $\mathcal{C}$. In more detail, we describe the topology of $\mathcal{G}$ by means of a topological graph $G_{\mathcal{G}}$, i.e. a graph isotopic to the curve. Then the topology of $\mathcal{C}$ is described by means of another graph $G_{\mathcal{C}}$ whose vertices are the images of the vertices of $G_{\mathcal{G}}$ under $\mathbf{x}$, and whose edges correspond to the branches of $\mathbf{x}(\mathcal{G})$, which are in one-to-one correspondence with the edges of $G_{\mathcal{G}}$. If $\mathbf{x}$ becomes infinite at a vertex of $G_{\mathcal{G}}$, the image of such a vertex corresponds to a branch at infinity of $\mathcal{C}$.

Additionally, the pair $\mathbf{x}, \mathcal{G}$ may come for free, or almost for free, in certain applications; see for instance the introductory example of an intersection curve at the beginning of Section 2. If the pair $\mathbf{x}, \mathcal{G}$ is known, in order to determine the topology of $\mathcal{C}$ one might compute an implicit representation 
of $\mathcal{C}$ using elimination methods. This yields one implicit equation in the plane case, and at least two implicit equations in the space case. In both cases, plane and space, after computing the implicit equation(s) one might use existing algorithms to find the topology of the curve: see for instance 17, 13, 17, 21], among many others, for the planar case, or [5, 12, 14, 18] for the space case. However, such an implicit representation typically has a high degree and big coefficients, which makes it difficult to use. Moreover, many algorithms have additional assumptions, for example generic position, or complete intersection in the space case, that are computationally expensive to fulfill. As a consequence, if the pair $\mathbf{x}, \mathcal{G}$ is known, it is useful to have an alternative method for computing the topology of $\mathcal{C}$ that avoids using an implicit representation.

On the other hand, if $\mathcal{C}$ is defined by means of an implicit representation the pair $\mathbf{x}, \mathcal{G}$ can be computed using a computer algebra system. Thus, our algorithm is applicable to that case as well, and provides an alternative to existing algorithms for computing the topology of a plane or space curve. This is specially useful in the space case, since known algorithms to compute the topology of a space case are not so easy to use in practice, and have a high complexity (see Section 6.3).

It is worth comparing our paper with some other related papers. In [4] the topology of 2D and 3D rational curves is addressed. In [4] the curve is seen as the image of the real line under a planar or space birational mapping, so somehow the germ of the idea in this paper is already in [4]. In [11], a method to compute the topology of a (non-necessarily rational) offset curve of a rational planar curve is provided. The method exploits similar ideas to [4], but focuses on offset curves, which have special properties. Finally, in [8] the problem of approximating a hyperelliptic curve by means of rational curves is considered. The Weierstrass form is also used in [8], but the goal is different, and in particular the computation of the topology of the hyperelliptic curve is not addressed.

Our method has been implemented in the computer algebra system Maple 2017, and the implementation can be freely downloaded from [29]. In order to certify the topology we need to certify self-intersections, i.e., we need to certify whether or not the image of two points under the birational mapping giving rise to our curve, is the same. This requires to work with algebraic numbers, and is computationally difficult. We address this problem, and we provide a complexity analysis of the algorithm with and without the certification step. While the complexity bound that we get is not better than the 
known complexity for the implicit planar case [24], it is, however, definitely better compared to the implicit space case [15, 12. It is true, however, that in 15, 12 the space curve is assumed to be given by an implicit representation. However, in our paper, even though the algorithm is applicable also to implicit curves after computing a Weierstrass form of the curve (which is efficient and fast), we assume a different representation of the curve, namely as the birational image of a Weierstrass curve.

The structure of this paper is the following. We motivate and present the problem in Section 2, where some preliminary notions and ideas are given. The planar case is addressed in Section 3, and the space case is studied in Section 4. In Section 5 we report on the results of our experimentation, carried out in the computer algebra system Maple 2017; we refer the interested reader to the ArXiv version of the paper [3] for the parametrizations used in the experimentation section. In Section 6, we address the complexity of the algorithm, we consider certification issues, and we compare the complexity of our algorithm with the known complexities of algorithms using an implicit representation of the curve. Section 7 contains our conclusions. The proofs of some results in Section 3 are postponed to Appendix I, so as not to stop the flow of the paper.

Acknowledgments: the authors want to thank the reviewers of the paper for their comments, which helped improve the quality of the paper.

\section{Motivation and presentation of the problem.}

Consider a biquadratic patch $S$, commonly used in Computer Aided Geometric Design, parametrized by

$$
\mathbf{x}(t, s)=(x(t, s), y(t, s), z(t, s))=\sum_{i=0}^{2} \sum_{j=0}^{2} \mathbf{c}_{i j} B_{i}(t) B_{j}(s)
$$

where $B_{k}(u)=\left(\begin{array}{l}2 \\ k\end{array}\right) u^{k}(1-u)^{2-k}$ for $k=0,1,2$, and $\mathbf{c}_{i j} \in \mathbb{R}^{3}$ for $i, j=0,1,2$. Assume that we want to describe the topology of the intersection curve $\mathcal{C}$ of $S$ with a general plane $\Pi$ of equation $A x+B y+C z+D=0$, i.e. the topology of $S \cap \Pi$. In order to do this, substituting the components $x(t, s)$, $y(t, s), z(t, s)$ of $\mathbf{x}(t, s)$ into the equation of $\Pi$ we get an algebraic condition $g(t, s)=0$; since the components of $\mathbf{x}(t, s)$ have bidegree $(2,2)$, one can see that

$$
g(t, s)=\Psi_{1}(t) s^{2}+\Psi_{2}(t) s+\Psi_{3}(t)=0,
$$


where the $\Psi_{i}(t), i=1,2,3$, are polynomials in the variable $t$. Then the curve $\mathcal{C}=S \cap \Pi$ can be described as the closure of the image of the planar curve $\mathcal{G}$, defined by $g(t, s)=0$ in the $(t, s)$-plane, under the (rational) mapping $\mathbf{x}$, i.e. $\mathcal{C}=\overline{\mathbf{x}(\mathcal{G})}$. Notice that $\mathcal{C}-\mathbf{x}(\mathcal{G})$ reduces to finitely many points corresponding to either the image of points of $\mathcal{G}$ at infinity, or limit points in $\mathcal{C}$ corresponding to base points of $\mathbf{x}$, lying in $\mathcal{G}$.

The situation presented above is an example of the general problem treated in this paper. Given a planar curve $\mathcal{G}$, implicitly defined in the plane $(t, s)$ by a polynomial equation like Eq. (2), of degree 2 in the variable $s$, our goal is to compute the topology of the curve $\mathcal{C}=\overline{\mathbf{x}(\mathcal{G})}$, where $\mathbf{x}: \mathbb{R}^{2} \rightarrow \mathbb{R}^{n}$, with $n=2$ or $n=3$, is birational when restricted to $\mathcal{G}$; in particular, in that case the inverse mapping $\left.\mathbf{x}\right|_{\mathcal{G}} ^{-1}: \mathcal{C} \rightarrow \mathcal{G}$ exists and is rational. Writing

$$
\mathbf{x}=\left(x_{1}, x_{2}, \ldots, x_{n}\right),
$$

we will refer to the functions $x_{i}: \mathbb{R}^{2} \rightarrow \mathbb{R}$ as the components of the mapping $\mathbf{x}$. Notice that if $\mathcal{C}$ is a rational curve, in which case the curve $\mathcal{G}$ must also be rational because of the birationality of the mapping $\left.\mathbf{x}\right|_{\mathcal{G}}$, then the problem can be solved using already existing methods [4]. Thus, we will assume that $\mathcal{C}$, and therefore also $\mathcal{G}$, is not rational, in which case $\mathcal{C}$ is said to be a hyperelliptic curve.

With some generality (see for instance [8]), we say that a curve $\mathcal{C}$ is $h y$ perelliptic if there exists a generically two-to-one map $\mathcal{C} \rightarrow \mathbb{R}$. Furthermore, such a curve (see for instance [26]) is birationally equivalent to a planar curve

$$
s^{2}-p(t)=0
$$

where $p(t)$ is a square-free polynomial of degree $2 \mathbf{g}+1$ or $2 \mathbf{g}+2$, where $\mathbf{g}$ is the genus of $\mathcal{C}$. Recall (see for instance [28]) that the genus $\mathbf{g}$ is a birational invariant that, in particular, characterizes rational curves: $\mathbf{g}=0$ corresponds to rational curves, while for non-rational curves $\mathbf{g} \geq 1, \mathbf{g} \in \mathbb{N}$. Additionally, whenever we work over a field of characteristic different from 2 , as it is our case, one can always get a Weierstrass curve where the degree of $p(t)$ is $2 \mathbf{g}+1$ (see for instance [26]). Also, Eq. (3) is called the Weierstrass form of $\mathcal{C}$. Notice (see p. 59 of [8]) that we can always transform the expression Eq. (2) of our motivating example into an expression like Eq. (3) by considering a change of parameters

$$
t:=t, s:=\frac{-B(t)+s}{2 A(t)}
$$


In this paper we will assume that the Weierstrass form has already been computed, and therefore that the curve $\mathcal{G}$ is described by means of Eq. (3). Additionally, we will assume that the curve $\mathcal{G}$ is real, i.e. that it contains infinitely many real points; if $\mathcal{G}$ is not real, then because of the birationality of $\left.\mathbf{x}\right|_{\mathcal{G}}, \mathcal{C}$ cannot be real either. Observe also that since $s^{2}-p(t)$ is an irreducible polynomial in $t, s$, so is the curve $\mathcal{G}$; since irreducibility is a birational invariant, we deduce that $\mathcal{C}$ is irreducible as well.

In order to describe the topology of the curve $\mathcal{C}$, we will compute, as it is common, a graph isotopic to $\mathcal{C}$.

Definition 1. Let $X, Y \subset \mathbb{R}^{n}$. We say that $X, Y$ are isotopic if there exists a continuous map $H: X \times[0,1] \rightarrow \mathbb{R}^{n}$ satisfying the following conditions: (1) $H(\bullet ; 0)$ is the identity; (2) $H(X ; 1)=Y$; (3) for all $\omega \in[0,1], H(\bullet ; \omega)$ is a homeomorphism from $X$ to $H(X: \omega)$. In this case, $H$ is called an isotopy between $X, Y$.

If $X, Y$ in Definition 1 are 1-dimensional objects, the fact that $X, Y$ are isotopic implies that one of them can be deformed into the other without removing or introducing self-intersections (see for instance [22]). Now we have the following definition.

Definition 2. Let $\mathcal{C} \subset \mathbb{R}^{n}$, where $n=2$ or $n=3$. A topological graph of $\mathcal{C}$ is a graph $G_{\mathcal{C}}$ isotopic to $\mathcal{C}$ whose vertices lie on the curve $\mathcal{C}$.

Remark 1. Vertices of $G_{\mathcal{C}}$ with valence equal to one, i.e. belonging only to one edge, correspond to real branches of $\mathcal{C}$ at infinity. Thus, if $G_{\mathcal{C}}$ contains some vertex of this type, then $\mathcal{C}$ is not bounded.

Thus, our goal is to build an algorithm for computing a topological graph $G_{\mathcal{C}}$ of $\mathcal{C}$; we will refer to $G_{\mathcal{C}}$ as the graph associated with $\mathcal{C}$. In order to do this, we will not compute $G_{\mathcal{C}}$ directly: instead, we will compute a graph $G_{\mathcal{G}}$ associated with $\mathcal{G}$, and we will derive $G_{\mathcal{C}}$ from $G_{\mathcal{G}}$ by studying how the topology of $\mathcal{G}$ changes when $\mathrm{x}$ is applied. Furthermore, in our analysis we do not consider isolated real points of $\mathcal{C}$, which can be generated by complex branches of $\mathcal{G}$ at infinity. Let us briefly recall how graphs associated with planar and space curves are computed.

\section{GRAPH ASSOCIATED WITH A PLANAR CURVE.}

Let $f(x, y)=0$ define a planar algebraic curve $\mathcal{F}$ without vertical asymptotes. We say that $P \in \mathcal{F}$ is regular if either $f_{x}(P) \neq 0$ or $f_{y}(P) \neq 0$; 
otherwise, we say that $P$ is singular. We say that $P \in \mathcal{F}$ is critical if $P$ satisfies that $f(P)=f_{y}(P)=0$. A critical point which is not singular is called a ramification point. The topological graph $G_{f}$ associated with $\mathcal{F}$ can be described as follows (see Fig. 1, left):

- The vertices of the graph $G_{f}$ are: $(1)$ the critical points of $\mathcal{F} ;(2)$ the points of $\mathcal{F}$ lying on the vertical lines through the critical points of $\mathcal{F}$ (we call these vertical lines, critical lines); (3) the points of $\mathcal{F}$ lying on vertical lines placed: (3.1) between two consecutive critical lines, (3.2) at the left of the left-most critical point, and (3.3) at the right of the right-most critical point.

- Two vertices of $G_{f}$ are connected by an edge of $G_{f}$ iff there is a real branch of $\mathcal{F}$ connecting the corresponding points on $\mathcal{F}$.

The problem of computing a topological graph of an implicit planar curve is well-studied. The interested reader can check the references [7, 13, 17, 21], among others, for further information on the problem. Although it is customary, in most papers dealing with the problem of computing the graph $G_{f}$, to start with the assumption that $\mathcal{F}$ does not have vertical asymptotes or vertical components, one can adapt the strategy without assuming these properties; see for instance [6].

\section{GRAPH ASSOCIATED WITH A SPACE CURVE.}

Let $\left\{f_{1}(x, y, z)=0, \ldots, f_{m}(x, y, z)=0\right\}$ define a space algebraic curve $\mathcal{F}$ : (i) without asymptotes parallel to the $z$-axis; (ii) such that the projection $\pi_{x y}(\mathcal{F})$ of $\mathcal{F}$ onto the $x y$-plane is birational. Hypothesis (iii) ensures that there are not two different real branches of $\mathcal{F}$ projecting onto a same branch of $\pi_{x y}(\mathcal{F})$. Taking advantage of Hypothesis (ii), the usual strategy to compute a topological graph $G_{f}$ isotopic to $\mathcal{F}$ is to birationally project $\mathcal{F}$ onto some plane, say, the $x y$-plane, then compute a graph isotopic to the projection $\pi_{x y}(\mathcal{F})$, which is a planar algebraic curve, and later "lift" the graph associated with $\pi_{x y}(\mathcal{C})$ to a space graph: this is the strategy followed in papers like 12, 14, 18, and we will follow this strategy here as well. Since the projection $\pi_{x y}$ is birational, one can be sure that every edge of the graph associated with $\pi_{x y}(\mathcal{F})$ lifts to one, and just one, edge of the graph associated with $\mathcal{F}$. More precisely, the graph $G_{f}$ associated with $\mathcal{F}$ can be described as follows (see Fig. 1, right): 

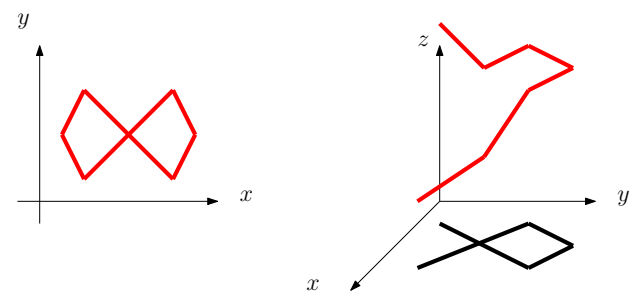

Figure 1: Graphs associated with planar and space curves

- The vertices of the graph $G_{f}$ are the points of $\mathcal{F}$ projecting as vertices of the graph associated with $\pi_{x y}(\mathcal{F})$.

- Two vertices of $G_{f}$ are connected by an edge of $G_{f}$ iff the corresponding points of $\mathcal{C}$ are connected by a real branch of $\mathcal{C}$. Furthermore, if the vertices are not singularities of $\pi_{x y}(\mathcal{C})$, we connect them iff their projections are connected in the graph associated with $\pi_{x y}(\mathcal{F})$. For vertices corresponding to singularities of $\pi_{x y}(\mathcal{C})$ the process is more complicated, since we can have two non-overlapping branches of $\mathcal{C}$ whose projections onto the $x y$-plane overlap (see Fig. 1, left); for references on how to deal with this problem, one can check [14, 18].

The problem of computing a topological graph associated with an implicit space algebraic curve has received some attention in the literature, although less than the planar case. The interested reader can check the references [5, 12, 14, 18] for more details on the problem. Again, as it also happens in the planar case, the strategy can be adapted to the case when $\mathcal{F}$ has vertical components or vertical asymptotes.

IN OUR CASE.

In our case, we need to compute the graph $G_{\mathcal{G}}$ associated with $\mathcal{G}$ plus some extra vertices $Q_{i}=\left(t_{i}, s_{i}\right) \in \mathcal{G}$. In particular, we need to include points $Q_{i} \in \mathcal{G}$ giving rise to certain notable points $P_{i} \in \mathcal{C}$, as we will see in the next sections. And we also need to include the points $Q_{i} \in \mathcal{G}$ where some component of $\mathbf{x}$ has the indeterminacy $\frac{0}{0}$, or becomes infinite. After including these vertices, we observe that $\mathbf{x}$ is continuous over each portion of the curve $\mathcal{G}$ corresponding to each edge of $G_{\mathcal{G}}$. Then, the key idea is that since the image of any connected subset of $\mathcal{G}$ is also connected, every edge $e$ of $G_{\mathcal{G}}$ gives rise to an edge $\tilde{e}$ of $G_{\mathcal{C}}$, namely the edge connecting the images of the 
vertices of $e$. Hence, the topology of $\mathcal{G}$ guides us to compute the topology of $\mathcal{C}$.

The fact that $\mathbf{x}$ is birational over $\mathcal{G}$ guarantees that all the edges of $G_{\mathcal{C}}$ are obtained this way, since there cannot be any real branch of $\mathcal{C}$ coming from a complex branch of $\mathcal{G}$ : indeed, if $\mathcal{B} \subset \mathcal{G}$ is a complex branch such that $\mathbf{x}(\mathcal{B})$ is real, then $\mathbf{x}(\mathcal{B})=\overline{\mathbf{x}(\mathcal{B})}$, where $\overline{\mathbf{x}(\mathcal{B})}$ denotes the conjugate of $\mathbf{x}(\mathcal{B})$. But then there are infinitely many points of $\mathcal{C}$ with at least two pre-images, which cannot happen because $\left.\mathbf{x}\right|_{\mathcal{G}}$ is birational.

Therefore, the rough idea in order to build $G_{\mathcal{C}}$ is to compute the graph $G_{\mathcal{G}}$ (by using any of the well-known algorithms to do this), and the images $P_{i}$ of the vertices $V_{i}$ of $G_{\mathcal{G}}$. Then we connect the $P_{i}$ according to how their preimages $V_{i}=\left.\mathbf{x}\right|_{\mathcal{G}} ^{-1}\left(P_{i}\right)$ are connected in $\mathcal{G}$. If some component of $\mathbf{x}\left(V_{i}\right)$ becomes infinite, then we have an open branch of $\mathcal{C}$, i.e. a branch of $\mathcal{C}$ going to infinity; in particular, in that case $\mathcal{C}$ is not bounded.

Fig. 2 represents the idea of computing $G_{\mathcal{C}}$ from $G_{\mathcal{G}}$, for the case $n=2$ : each edge, marked with a different color, of the graph $G_{\mathcal{G}}$ (left), gives rise to an edge, marked with the same color, of the graph $G_{\mathcal{C}}$ (right).

Observe that since $\mathcal{G}$ is implicitly defined by Eq. (3), the leading coefficient in the variable $s$ is constant, so $\mathcal{G}$ has no asymptotes parallel to the $s$-axis, which we take as the vertical axis in the $(t, s)$ plane. Additionally, since the Weierstrass form implies that $p(t)$ is square-free, one can see that $\mathcal{G}$ is regular, and that the only critical points are the points $\{s=0, p(t)=0\}$, all of which are ramification points, i.e. points where the tangent line to $\mathcal{G}$ is vertical. Because of this, $\mathcal{G}$ consists of open branches and/or closed components, without self-intersections. As a projective variey, though, $\mathcal{G}$ has a singular point, namely the point at infinity of $\mathcal{G}$ (in the direction of the $s$-axis).

Certainly, there can also be some points of $\mathcal{C}$ which do not belong to $\mathbf{x}(\mathcal{G})$. The points in $\mathcal{C}-\mathbf{x}(\mathcal{G})$ correspond to the images of the point at infinity of $\mathcal{G}$, and the limit points coming from the base points of $\mathbf{x}$ lying in $\mathcal{G}$, i.e. points of $\mathcal{G}$ where all the numerators and denominators of the components of $\mathbf{x}$ vanish simultaneously. Since $\mathcal{G}$ is regular over its affine part, we can be sure that $\mathbf{x}$ extends to its base points (see Theorem 1.2 of [23]), so that base points give rise to either affine points of $\mathcal{C}$, or points at infinity of $\mathcal{C}$. The effective computation of the images of base points of $\mathbf{x}$ on $\mathcal{G}$ is analyzed in the next section. On the other hand, $\mathcal{G}$ has one singular point at infinity with two different branches, i.e. two different places centered at this point (see 31 for further information on places). This implies that the point at 


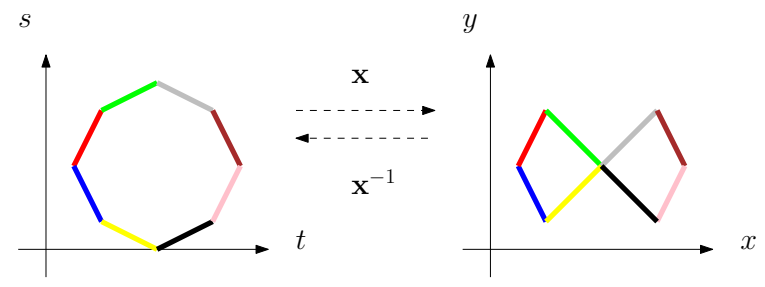

Figure 2: $G_{\mathcal{G}}$ and $G_{\mathcal{C}}$

infinity of $\mathcal{G}$ can give rise to at most two points of $\mathcal{C}$, that can be affine, or at infinity. We denote these points by $P_{\infty}, P_{-\infty}$, that may or may not coincide. This notation responds to the fact that these points are reached by analyzing the behavior of $\left.\mathbf{x}\right|_{\mathcal{G}}$ when $t \rightarrow \infty$ and $t \rightarrow-\infty$. In the next section, we will consider the computation of these points, that we will represent in a more compact way by $P_{ \pm \infty}$.

\section{The planar case.}

Let $\mathbf{x}: \mathbb{R}^{2} \rightarrow \mathbb{R}^{2}$, where

$$
\mathbf{x}(t, s)=(x(t, s), y(t, s))=\left(\frac{A_{1}(t, s)}{B_{1}(t, s)}, \frac{A_{2}(t, s)}{B_{2}(t, s)}\right),
$$

and let $\mathcal{C}=\mathbf{x}(\mathcal{G})$, where $\mathcal{G}$ is implicitly defined by an equation $g(t, s)=$ $s^{2}-p(t)=0$ like Eq. (3). The functions $x(t, s), y(t, s)$ are the components of $\mathbf{x}(t, s)$. We require $\mathbf{x}$ to be a rational mapping satisfying that the restriction $\left.\mathbf{x}\right|_{\mathcal{G}}$ is birational, so that $\left.\mathbf{x}\right|_{\mathcal{G}} ^{-1}: \mathcal{C} \rightarrow \mathcal{G}$ is well-defined, and therefore rational. We can always check this assumption with a probabilistic algorithm; we take a random point $\left(t_{0}, s_{0}\right) \in \mathcal{G}$, compute the point $P=\mathbf{x}\left(t_{0}, s_{0}\right)$, and finally determine the preimages of $\mathbf{x}\left(t_{0}, s_{0}\right)$ : if we get only one preimage belonging to $\mathcal{G}$, then with probability one the required hypothesis holds. Additionally, using repeatedly the fact that $s^{2}=p(t)$, we can write $\left.\mathbf{x}\right|_{\mathcal{G}}(t, s)$ in the following form:

$$
\left.\mathbf{x}\right|_{\mathcal{G}}(t, s)=\left(\frac{A_{1}(t, s)}{B_{1}(t, s)}, \frac{A_{2}(t, s)}{B_{2}(t, s)}\right)=\left(\frac{a_{11}(t)+s a_{12}(t)}{b_{11}(t)+s b_{12}(t)}, \frac{a_{21}(t)+s a_{22}(t)}{b_{21}(t)+s b_{22}(t)}\right),
$$

where we can assume that $A_{i}, B_{i}$ are relatively prime for $i=1,2$. Observe that this implies $\operatorname{gcd}\left(a_{11}, a_{12}, b_{11}, b_{12}\right)=1$ and $\operatorname{gcd}\left(a_{21}, a_{22}, b_{21}, b_{22}\right)=1$. Notice also that in general $b_{11}(t) \neq b_{21}(t), b_{12}(t) \neq b_{22}(t)$. 
As observed in Section 2, we first need to describe the topology of $\mathcal{G}$ by means of a graph $G_{\mathcal{G}}$ isotopic to it, with some additional vertices. We need to include the following points as vertices of $G_{\mathcal{G}}$ :

(i) Critical points of $g(t, s)=0$, i.e. points of $\mathcal{G}$ where $g_{s}=0$.

(ii) Points of $\mathcal{G}$ giving rise to critical points of $\mathcal{C}$.

(iii) Points of $\mathcal{G}$ where some component of $\mathbf{x}$ is not defined.

(iv) Starting and ending points for open branches of $\mathcal{G}$.

The points in (i) are the solutions of $g=g_{s}=0$, i.e. the points $\{s=$ $0, p(t)=0\}$. The points in (iv) can be easily computed by taking a $t$-value at the left (resp. right) of the left-most (resp. the right-most) solution of $g=g_{s}$. The points in (iii) are the points $(t, s) \in \mathcal{G}$ such that $B_{1}(t, s) \cdot B_{2}(t, s)=0$. In particular, some of the points in (iii) may generate asymptotes of $\mathcal{C}$; also, base points of $\mathbf{x}$ in $\mathcal{G}$, i.e. the points of $\mathcal{G}$ where

$$
A_{1}(t, s)=B_{1}(t, s)=A_{2}(t, s)=B_{2}(t, s)=g(t, s)=0,
$$

are included in (iii). The topology of $\mathcal{G}$ is easy to capture (see for instance [8), and can be computed by using known algorithms for planar curves like [7, 13, 17, 21].

\subsection{Computing the points of $\mathcal{G}$ giving rise to critical points of $\mathcal{C}$}

For simplicity, in this section we will assume that $\mathbf{x}$ has no base points on $\mathcal{G}$. These points, which may also generate critical points of $\mathcal{C}$, will be analyzed in the next subsection. Some observations on how to use the results in this subsection in the presence of base points will be done at the end of the subsection. Additionally, if the points $P_{ \pm \infty}$ are affine they may be critical points of $\mathcal{C}$ as well. The behavior of $P_{ \pm \infty}$ will be studied in Subsection 3.3 .

Now in Section 2 we recalled that the critical points of $\mathcal{C}$ are either singularities, or ramification points, i.e. points where the tangent line is vertical. It is useful to distinguish two types of singularities: local singularities, which correspond to singular points $P \in \mathcal{C}$ with just one branch of $\mathcal{C}$ through $P$, and self-intersections of $\mathcal{C}$, which correspond to points $P \in \mathcal{C}$ with at leat two different branches of $\mathcal{C}$ through $P$. In Fig. 3 we show three examples of local singularities, two of them cuspidal (first two curves, starting from the 

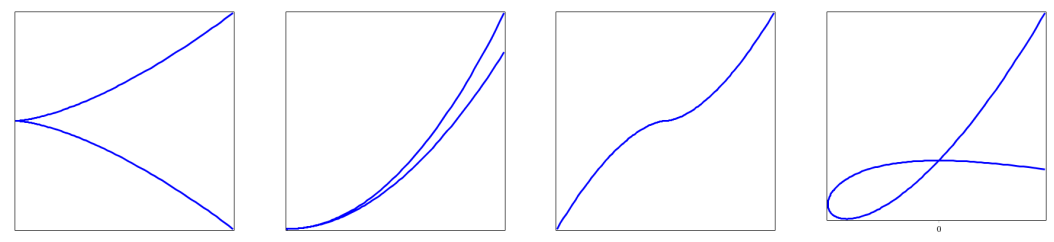

Figure 3: Local singularities (three local singularities and q self-intersection (right-most curve).

left) and one of them non-cuspidal (third curve, starting from the left), and a self-intersection (right-most curve); see [1] for more information on local singularities.

In order to compute the points of $\mathcal{G}$ giving rise to local singularities and ramification points of $\mathcal{C}$, we analyze $\mathbf{x}(\mathcal{G})$, where $\mathcal{G}$ is implicitly defined by $g(t, s)=0$. The differential of $\mathbf{x}$ defines a mapping between the tangent space to $\mathcal{G}$ and the tangent space to $\mathcal{C}$, at corresponding points. Denoting a generic element of the tangent space to $\mathcal{C}$ by $\boldsymbol{v}=\left(v_{1}, v_{2}\right)$, we have the following relationship; here, $x_{t}$ represents the partial derivative of $x(t, s)$ with respect to the variable $t$, and similarly for $y_{t}, x_{s}, y_{s}, g_{t}, g_{s}$ :

$$
\left[\begin{array}{ll}
x_{t} & x_{s} \\
y_{t} & y_{s}
\end{array}\right] \cdot\left[\begin{array}{c}
g_{s} \\
-g_{t}
\end{array}\right]=\left[\begin{array}{l}
v_{1} \\
v_{2}
\end{array}\right]
$$

The above relationship follows from differentiating with respect to $t$ the components of $\left.\mathbf{x}\right|_{\mathcal{G}}$. Whenever $g_{s} \neq 0$ (i.e. whenever $(t, s)$ is not a ramification point of $\mathcal{G}), g(t, s)=0$ implicitly defines a differentiable function $s=s(t)$, where $\frac{d s}{d t}=-\frac{g_{t}}{g_{s}}$. Now differentiating $\mathbf{x}(t, s)=0$ where $s=s(t)$ is the function defined by $g(t, s)=0$, and using the Chain Rule, we get a vector $\boldsymbol{w}$ which is parallel to the vector $\boldsymbol{v}$ in Eq. (5). For the points where $g_{s}=0$, we can proceed in the same way, reaching the same result, differentiating with respect to $s$ instead. Since all affine points of $\mathcal{G}$ are regular, i.e. either $g_{t}$ or $g_{s}$ are nonzero, Eq. (5) holds.

Lemma 3. Suppose that $\mathbf{x}$ has no base points lying on $\mathcal{G}$, and let $P \in \mathcal{C}$, $P \neq P_{ \pm \infty}, P=\mathbf{x}\left(t_{0}, s_{0}\right)$, where $\left(t_{0}, s_{0}\right) \in \mathcal{G}$. If $P$ is a either a local singularity or a ramification point of $\mathcal{C}$, then $\left(t_{0}, s_{0}\right)$ satisfies that

$$
g=x_{t} g_{s}-x_{s} g_{t}=0
$$


Remark 2. For the local singularities we have

$$
g=x_{t} g_{s}-x_{s} g_{t}=y_{t} g_{s}-y_{s} g_{t}=0 .
$$

However, Lemma 3 does not necessarily provide the self-intersections of $\mathcal{C}$. In order to find these last singularities, we imitate the strategy in [2]. First we define

$$
\begin{aligned}
\xi_{1}(x, t) & =\text { square-free part of } \operatorname{Res}_{s}(\operatorname{num}(x-x(t, s)), g(t, s)), \\
\xi_{2}(x, y, t) & =\text { square-free part of } \operatorname{Res}_{s}(\operatorname{num}(x-x(t, s)), \operatorname{num}(y-y(t, s))),
\end{aligned}
$$

where num $(\bullet)$ denotes the numerator of the rational function $\bullet$. Notice that in general, eliminating $t$ in $\xi_{1}(x, t)=0, \xi_{2}(x, y, t)=0$ by means of the resultant $\operatorname{Res}_{t}\left(\xi_{1}(x, t), \xi_{2}(x, y, t)\right)$, we obtain a polynomial in $x, y$ containing, as a factor, the implicit equation of $\mathcal{C}$. Using the definition of the resultant, one can easily check that $\xi_{1}(x, t)$ is a quadratic polynomial in $x$, and $\xi_{2}(x, y, t)$ is quadratic as a polynomial in $x, y$, and linear in $x$ and in $y$ (i.e. $\xi_{2}(x, y, t)$ is bilinear).

Now the key idea to find the self-intersections of $\mathcal{C}$ is that these points are among the points $(x, y) \in \mathcal{C}$ where $t=\left.\mathbf{x}\right|_{\mathcal{G}} ^{-1}(x, y)$ is not defined. For a generic point $\left(x_{0}, y_{0}\right) \in \mathcal{C}$, we can find $t_{0}=\left.\mathbf{x}\right|_{\mathcal{G}} ^{-1}\left(x_{0}, y_{0}\right)$ as the only root of $\operatorname{gcd}\left(\xi_{1}\left(x_{0}, t\right), \xi_{2}\left(x_{0}, y_{0}, t\right)\right)$. In order to find the function $t=t(x, y)=$ $\left.\mathbf{x}\right|_{\mathcal{G}} ^{-1}(x, y)$, we can compute the gcd of $\xi_{1}(x, t)$ and $\xi_{2}(x, y, t)$ as polynomials in the variable $t$ whose coefficients are real polynomials in $x, y$, with the additional condition $f(x, y)=0$, where $f$ is the implicit equation of $\mathcal{C}$. More formally, one sees $\xi_{1}(x, t)$ and $\xi_{2}(x, y, t)$ as elements of $\mathbb{R}(\mathcal{C})[t]$, where $\mathbb{R}(\mathcal{C})$ is the field of real rational functions of $\mathcal{C}$. Since $\mathcal{C}$ is irreducible $\mathbb{R}(\mathcal{C})$ is a Euclidean domain. Therefore

$$
D(x, y, t)=\underset{\mathbb{R}(\mathcal{C})[t]}{\operatorname{gcd}}\left(\xi_{1}, \xi_{2}\right)
$$

is well-defined and can be computed, for instance, by means of the Euclidean algorithm. Since $\left.\mathbf{x}\right|_{\mathcal{G}}$ is proper, $D(x, y, t)$ is linear in $t$ and solving $D(x, y, t)=$ 0 for $t$, one gets $t=\left.\mathbf{x}\right|_{\mathcal{G}} ^{-1}(x, y)$.

Following the ideas of [2], one can compute $\left.\mathbf{x}\right|_{\mathcal{G}} ^{-1}(x, y)$ more eficiently as follows (see [2] for further detail). By the fundamental property of subresultants, $D(x, y, t)$ is the first subresultant different from zero (modulo $f(x, y)$ ) in the subresultant chain of $\xi_{1}, \xi_{2}$, seen as elements of the domain $\mathbb{R}[x, y][t]$. 
If the degrees of $\xi_{1}, \xi_{2}$ as elements of $\mathbb{R}[x, y][t]$ are $n_{1}, n_{2}$, the elements of the subresultant chain are represented as

$$
\left\{\operatorname{Subres}_{i}\left(\xi_{1}, n_{1}, \xi_{2}, n_{2}\right)_{i \geq 0}\right\},
$$

with $0 \leq i \leq \inf \left(n_{1}, n_{2}\right)-1$, and can be defined as determinants of order $n_{1}+n_{2}-i$ of Sylvester-like matrices whose entries are related to the coefficients of $\xi_{1}, \xi_{2}$ (see Section 2.2 of [2]). Since $\operatorname{deg}\left(\operatorname{Subres}_{i}\left(\xi_{1}, n_{1}, \xi_{2}, n_{2}\right)\right) \leq i$, and by the birationality of $\left.\mathbf{x}\right|_{\mathcal{G}}$ we have $\operatorname{deg}\left(G\left(x_{0}, y_{0}, t\right)\right)=1$ for almost all $\left(x_{0}, y_{0}\right) \in \mathcal{C}$, we deduce that $D(x, y, t)$ is equal to $\operatorname{Subres}_{1}\left(\xi_{1}, n_{1}, \xi_{2}, n_{2}\right)$; notice that $\operatorname{Subres}_{1}\left(\xi_{1}, n_{1}, \xi_{2}, n_{2}\right)$ can be computed without actually knowing the implicit equation of $\mathcal{C}$. Writing

$$
\operatorname{Subres}_{1}\left(\xi_{1}, n_{1}, \xi_{2}, n_{2}\right)(t)=\operatorname{sres}_{1}(x, y) t+\operatorname{sr}_{1}(x, y),
$$

we have that

$$
t=\left.\mathbf{x}\right|_{\mathcal{G}} ^{-1}(x, y)=-\frac{\operatorname{sr}_{1}(x, y)}{\operatorname{sres}_{1}(x, y)} .
$$

The polynomial $\operatorname{sres}_{1}(x, y)$ is called the first principal subresultant of $\xi_{1}, n_{1}$ and $\xi_{2}, n_{2}$. Finally we get the following result.

Theorem 4. Suppose that $\mathbf{x}$ has no base points lying on $\mathcal{G}$, and let $P \in \mathcal{C}$, $P=\mathbf{x}\left(t_{0}, s_{0}\right), P \neq P_{ \pm \infty}$. If $P$ is a self-intersection, then $\left(t_{0}, s_{0}\right)$ is a solution of the bivariate polynomial system

$$
\operatorname{sres}_{1}(x(t, s), y(t, s))=0, g(t, s)=0 .
$$

The next result shows that, in fact, all the singularities of $\mathcal{C}$, i.e. the local singularities and the self-intersections, except perhaps for $P_{ \pm \infty}$, are solutions of Eq. (10). The proof of this result in given in Appendix I, so as not to stop the flow of the paper.

Proposition 5. Let $\left(t_{0}, s_{0}\right) \in \mathcal{G}$ be a point such that

$$
\left(x_{0}, y_{0}\right)=\left(x\left(t_{0}, s_{0}\right), y\left(t_{0}, s_{0}\right)\right) \in \mathcal{C}
$$

is not a self-intersection, with

$x_{t}\left(t_{0}, s_{0}\right) g_{s}\left(t_{0}, s_{0}\right)-x_{s}\left(t_{0}, s_{0}\right) g_{t}\left(t_{0}, s_{0}\right)=y_{t}\left(t_{0}, s_{0}\right) g_{s}\left(t_{0}, s_{0}\right)-y_{s}\left(t_{0}, s_{0}\right) g_{t}\left(t_{0}, s_{0}\right)=0$.

\section{Then $\operatorname{sres}_{1}\left(x_{0}, y_{0}\right)=0$.}


Proposition 5 provides the following result.

Theorem 6. Suppose that $\mathbf{x}$ has no base points lying on $\mathcal{G}$. Then every singularity of $\mathcal{C}$, except perhaps for $P_{ \pm \infty}$, is a solution of Eq. (10).

The analysis of $P_{ \pm \infty}$ is postponed to Section 3.3. Additionally, there is another point missing in the discussion before. In order for the subresultant chain of $\xi_{1}, \xi_{2}$ not to vanish completely, we must require that $\xi_{1}, \xi_{2}$ do not share any factor depending on $t$. We identify the cases when this happens in the following two results. The proofs of these results are given in Appendix I.

Lemma 7. The polynomials $\xi_{1}(x, t)$ and $\xi_{2}(x, y, t)$ have a common factor $t-t_{0}$ iff $t_{0}$ corresponds to a base point of $\mathbf{x}$, lying on $\mathcal{G}$.

Lemma 8. The polynomials $\xi_{1}(x, t)$ and $\xi_{2}(x, y, t)$ have a common factor $\eta(x, t)$ depending on both $x, t$ iff $x(t, s)$ depends only on $t$.

In the case of Lemma 7, if $\mathbf{x}$ has some base point lying on $\mathcal{G}$ we remove the common factor depending on $t$, and perform the procedure presented before. In the case of Lemma 8, we replace $\xi_{2}(x, y, t)$ by

$$
\tilde{\xi}_{2}(y, t)=\text { square-free part of } \operatorname{Res}_{s}(\operatorname{num}(y-y(t, s)), g(t, s)) \text {, }
$$

and proceed as before.

\subsection{Behavior of $\mathcal{C}$ around the base points of $\left.\mathbf{x}\right|_{\mathcal{G}}$.}

Let $Q=\left(t_{0}, s_{0}\right) \in \mathcal{G}$ be a base point of $\left.\mathbf{x}\right|_{\mathcal{G}}$. Notice that by Lemma 7. $t=t_{0}$ must be a root of the content of $\xi_{1}, \xi_{2}$ with respect to $t$, and therefore has been previously determined. In this case, $\mathbf{x}\left(t_{0}, s_{0}\right)=\left(\frac{0}{0}, \frac{0}{0}\right)$. Although the fact that the $\mathcal{G}$ does not have affine singularities guarantees that $\mathbf{x}\left(t_{0}, s_{0}\right)$ is defined as a projective point (see Theorem 1.2 of [23]), we still need to determine the behavior of $\mathbf{x}$ when the point $\left(t_{0}, s_{0}\right)$ is approached; in particular, we need to check if we get an affine point or a point at infinity, in which case we get an infinite branch of $\mathcal{C}$. In order to do this, we distinguish two situations:

(i) The point $\left(t_{0}, s_{0}\right)$ is not a critical point of $\mathcal{G}$ : in this case, by the Implicit Function Theorem $s^{2}-p(t)=0$ implicitly defines $s=s(t)$ at $t=t_{0}$. 
In fact, we can easily find the Taylor expansion of the function $s(t)$ at $t=t_{0}$, and then study the limits

$$
\lim _{t \rightarrow t_{0}} x(t, s(t)), \lim _{t \rightarrow t_{0}} y(t, s(t)) .
$$

If both limits are finite, then $\left(t_{0}, s_{0}\right)$ generates an affine point of $\mathcal{C}$. Otherwise we have a branch going to infinity, which is an asymptote of $\mathcal{C}$ whenever one of the above limits is finite.

(ii) The point $\left(t_{0}, s_{0}\right)$ is a critical point of $\mathcal{G}$ : in this case $t_{0}$ is a root of $p(t)$, so $s_{0}=0$. Now we consider $s= \pm \sqrt{p(t)}$ and we study each branch $s=\sqrt{p(t)}$ and $s=-\sqrt{p(t)}$ separately. We address in more detail the case $s=\sqrt{p(t)}$; for $s=-\sqrt{p(t)}$ the analysis is similar. Now if $s=\sqrt{p(t)}$, for the component $x(t, s)$ we have

$$
x(t, \sqrt{p(t)})=\frac{a_{11}(t)+\sqrt{p(t)} a_{12}(t)}{b_{11}(t)+\sqrt{p(t)} b_{12}(t)} .
$$

We are interested in analyzing the behavior of this function when $t \rightarrow t_{0}$. Since $\left(t_{0}, 0\right)$ is a base point of $x(t, s), a_{11}\left(t_{0}\right)=b_{11}\left(t_{0}\right)=0$. Additionally, since $a_{11}(t), a_{12}(t), b_{11}(t), b_{12}(t)$ are relatively prime, it cannot be $a_{12}\left(t_{0}\right)=0$ and $b_{12}\left(t_{0}\right)=0$ simultaneously. Furthermore, $t=t_{0}$ is a root of $p(t)$, and since $p(t)$ does not have multiple roots, the multiplicity of $t_{0}$ is 1 . Hence we can factor out $\left(t-t_{0}\right)^{1 / 2}$ in the numerator and denominator of $x(t, \sqrt{p(t)})$, and we get

$$
x(t, \sqrt{p(t)})=\frac{\tilde{a}_{11}(t)+\sqrt{\tilde{p}(t)} a_{12}(t)}{\tilde{b}_{11}(t)+\sqrt{\tilde{p}(t)} b_{12}(t)},
$$

where $\tilde{a}_{11}(t)=\frac{a_{11}(t)}{\left(t-t_{0}\right)^{1 / 2}}, \tilde{b}_{11}(t)=\frac{b_{11}(t)}{\left(t-t_{0}\right)^{1 / 2}}$, and $\tilde{p}(t)=\frac{p(t)}{t-t_{0}}$.

Observe that since $a_{11}\left(t_{0}\right)=b_{11}\left(t_{0}\right)=0$ and $a_{11}(t), b_{11}(t)$ are polynomials, $\tilde{a}_{11}\left(t_{0}\right)=\tilde{b}_{11}\left(t_{0}\right)=0$. Therefore, when $t \rightarrow t_{0}$ the limit of the function $x(t, \sqrt{p(t)})$ is equal to the limit of $a_{12}(t) / b_{12}(t)$ when $t \rightarrow t_{0}$. Since not both $a_{12}\left(t_{0}\right), b_{12}\left(t_{0}\right)$ are zero, the limit is defined whenever $b_{12}\left(t_{0}\right) \neq 0$, and is infinite (in which case we have a branch at infinity) whenever $b_{12}\left(t_{0}\right)=0$. Similarly for the component $y(t, s)$, and for $s=-\sqrt{p(t)}$. 
Notice that these ideas can be also used at points $\left(t_{0}, s_{0}\right)$ where only one component of $\left.\mathbf{x}\right|_{\mathcal{G}}(t, s)$ is undefined. Observe also that when working in a projective setting, the point at infinity of the curve $\mathcal{G},(0: 1: 0)$, which gives rise to $P_{ \pm \infty}$, is also a base point of the mapping $\mathbf{x}$ (see Eq. (40). The analysis of the behavior of the mapping $\mathbf{x}$ around this point is carried out in the next subsection.

\subsection{Computation and study of $P_{ \pm \infty}$.}

The point at infinity of the curve $\mathcal{G}$ is the center of two places, i.e. two branches of $\mathcal{G}$. In turn, these two branches generate two branches of $\mathcal{C}$ via $\mathbf{x}$, which can be centered at affine points or points at infinity denoted by $P_{ \pm \infty}$. In order to compute whether or not the $P_{ \pm \infty}$ are affine, we must study the (four) limits

$$
\lim _{t \rightarrow \pm \infty} \mathbf{x}(t, \sqrt{p(t)}), \quad \lim _{t \rightarrow \pm \infty} \mathbf{x}(t,-\sqrt{p(t)})
$$

Notice that we can have at most two different finite values in these limits, corresponding to the case when all $P_{ \pm \infty}$ are affine. In order to compute these limits, after performing elementary calculations we arrive to an expression $\frac{\mu_{1}(t)}{\mu_{2}(t)}$ where one of the $\mu_{i}(t)$ is a polynomial, and the other $\mu_{i}(t)$ involves polynomials and one radical term. Then the limit can be evaluated by just comparing the degrees of the numerator and the denominator; notice that the degree can be a non-integer, rational number in the case of the numerator or denominator involving a square-root. In our experimentation we have checked that a computer algebra system like Maple 18 perfectly computes these limits in almost no time.

It can happen that all $P_{ \pm \infty}$, only some of them, or none of them, is affine. If all $P_{ \pm \infty}$ are affine and equal, then $P_{ \pm \infty}$ is a self-intersection of $\mathcal{C}$. In this case, if the branches at infinity of $\mathcal{G}$ are real, then there are at least two real branches of $\mathcal{C}$ passing through $P_{ \pm \infty}$; if the branches are complex and $P_{ \pm \infty}$ is real, then $P_{ \pm \infty}$ is an isolated point of $\mathcal{C}$. If some $P_{ \pm \infty}$ is affine, it can also be a self-intersection of $\mathcal{C}$ when there exists an affine point of $\mathcal{G}$ whose image under $\mathbf{x}(t, s)$ coincides with this $P_{ \pm \infty}$. This can be checked by solving the bivariate system $\left\{\mathbf{x}(t, s)=P_{ \pm \infty}, g(t, s)=0\right\}$.

Additionally, when some of the $P_{ \pm \infty}$ are affine, we can check whether they are local singularities by checking whether the limit for $t \rightarrow \pm \infty$ of the derivative of $\mathbf{x}(t, \pm \sqrt{p(t)})$ vanishes. 


\subsection{Construction of $G_{\mathcal{C}}$.}

Let $Q_{1}=\left(t_{1}, s_{1}\right), \ldots, Q_{r}=\left(t_{r}, s_{r}\right)$ be the points of $\mathcal{G}$ computed in (i)(iv) (see the beginning of Section 3). Since the $Q_{i}$ belong to $\mathcal{G}$ and the graph associated with $\mathcal{G}$ can be computed by means of well-known methods [7, 13, 17, 21], we know how to connect the $Q_{i}$ to each other. Furthermore, from the preceding sections the behavior of $\mathbf{x}$ around the $Q_{i}$ is clear. Now the vertices of $G_{\mathcal{C}}$ are the images $P_{i}=\mathbf{x}\left(Q_{i}\right)$, whenever $\mathbf{x}\left(Q_{i}\right)$ (or the limit of $\mathbf{x}(t, s)$ as $(t, s) \rightarrow Q_{i}$, in the case of base points) is defined, and we connect two of these vertices iff their preimages $Q_{i}$ are connected to each other in $G_{\mathcal{G}}$. Furthermore, we also include as vertices of $G_{\mathcal{C}}$ the points $P_{ \pm \infty} \in \mathcal{C}$ coming from the point at infinity of $\mathcal{G}$, in case they are affine.

Additionally, the graph associated with $\mathcal{G}$ can have open edges (representing branches tending to infinity), corresponding to the edges of $\mathcal{G}$ with some vertex where some component of $\mathbf{x}$ becomes infinite, or branches of $\mathcal{G}$ tending to infinity, in the case when some $P_{ \pm \infty}$ is at infinity. Also, we must check that the edges of the graph associated with $\mathcal{C}$ do not intersect except at the self-intersections of $\mathcal{C}$. This is not impossible. However, we can check whether this happens by computing the number of self-intersections of the edges of the graph, and checking whether this number agrees with the number of self-intersections, which has been computed previously. Notice that in order to check whether two segments intersect it is not necessary to explicitly find the equations of the lines containing the segments, or solving a linear system of equations. It can be decided directly from the coordinates of the vertices, and is a usual operation in Computational Geometry, negligible in terms of computation time. If the number of crossings between the edges is higher than the number of self-intersections of $\mathcal{C}$, previously determined, we just introduce additional vertices in the graph until the spurious crossings are avoided. In the following theorem, we will assume that this test has been carried out, so that the number of self-intersections is correct.

Theorem 9. Let $G_{\mathcal{C}}$ be the graph associated with $\mathcal{C}$ according to the description in the preceding subsections. Then $G_{\mathcal{C}}$ and $\mathcal{C}$ are isotopic.

Proof. Once we compute the points of $\mathcal{G}$ where $\mathbf{x}$ becomes infinite, $\mathcal{G}$ is segmented into finitely many portions $\ell_{1}, \ldots, \ell_{p}$ where $\mathbf{x}$ is continuous. Each $\ell_{i}$ is connected, and by continuity $\mathbf{x}\left(\ell_{i}\right)$ is connected as well. Furthermore, by the birationality of $\left.\mathbf{x}\right|_{\mathcal{G}}$ the correspondence between the $\ell_{i}$ and the $\mathbf{x}\left(\ell_{i}\right)$ is $1: 1$. Since $\mathcal{C}=\mathbf{x}(\mathcal{G})$ and $\mathbf{x}(\mathcal{C})$ coincides with the union of the $\mathbf{x}\left(\ell_{i}\right)$, we just 
need to show that the graph $G_{\mathcal{C}}$ is isotopic to the union of the $\mathbf{x}\left(\ell_{i}\right)$. Since in $G_{\mathcal{C}}$ we are just deforming each $\mathbf{x}\left(\ell_{i}\right)$ into a segment, in order to show that $G_{\mathcal{C}}$ and $\mathcal{C}$ are isotopic we just need to show that no self-intersections of $\mathcal{C}$ are missed, and that no other self-intersections are introduced. The former is guaranteed by construction, since in the process of computing $G_{\mathcal{C}}$ all the self-intersections of $\mathcal{C}$ are identified. The latter is guaranteed by checking that two edges do not intersect at a point which is not a self-intersection of $\mathcal{C}$.

Example 1. Let

$$
g(t, s)=s^{2}+t^{4}-t^{3}-27 t^{2}+25 t+50=0,
$$

and let

$$
\mathbf{x}(t, s)=(x(t, s), y(t, s))=\left(\frac{t^{4}-t^{3}+t^{2}+5 s-t}{t^{6}+1}, \frac{t^{4}+t^{3}-t^{2}-5 s+t}{t^{6}+1}\right) .
$$

The curve $\mathcal{C}=\mathbf{x}(\mathcal{G})$ is a hyperelliptic curve of genus one.

First we compute the real points $(t, s) \in \mathcal{G}$ generating the vertices of $G_{\mathcal{C}}$ :

(i) Critical points of $g(t, s)=0$, i.e. points $(t, 0)$ with $p(t)=0$ :

$$
Q_{1}=(-5,0), Q_{2}=(-1,0), Q_{3}=(2,0) \text { and } Q_{4}=(5,0) \text {. }
$$

(ii) Points of $\mathcal{G}$ giving rise to critical points of $\mathcal{C}$. Local singularities and ramification points are generated by the points $(t, s)$ solutions of the system

$$
g(t, s)=0, \quad x_{t} g_{s}-x_{s} g_{t}=0 .
$$

The real solutions (written only with two digits) are:

$$
\begin{gathered}
Q_{5}=(-4.98,-2.05), Q_{6}=(-3.21,-13.00), Q_{7}=(-1.16,-3.47), \\
Q_{8}=(-1.12,3.08), Q_{9}=(2.15,3.11), Q_{10}=(2.24,-3.97) \\
Q_{11}=(3.76,-9.54), Q_{12}=(4.96,-2.52)
\end{gathered}
$$

Now we compute the points of $\mathcal{G}$ giving rise to self-intersections of $\mathcal{C}$. We have:

$$
\xi_{1}(x, t)=\left(t^{12}+2 t^{6}+1\right) x^{2}+\left(-2 t^{10}+2 t^{9}+\cdots\right) x+t^{8}+\cdots+1250,
$$


and

$$
\xi_{2}(x, y, t)=\left(t^{6}+1\right)(x+y)-2 t^{4} .
$$

The self-intersections of $\mathcal{C}$ are generated by the real solutions of the system $\left\{\operatorname{sres}_{1}(x(t, s), y(t, s))=0, \quad g(t, s)=0\right\}$, which are $Q_{13}=$ $(-3.75,-13.14), Q_{14}=(-2.32,-10.61), Q_{15}=(2.32,-4.62)$ and $Q_{16}=$ $(3.75,9.53)$.

The points $Q_{13}$ and $Q_{16}$ both generate the same point, $P_{13}$, and the points $Q_{14}$ and $Q_{15}$ both generate the point $P_{14}$ (see Figure 5 ).

(iii) Points of $\mathcal{G}$ where some component of $\mathbf{x}$ is not defined: there are neither base points nor vertical asymptotes.

(iv) Starting and ending points for open branches of $G$ : There are not open branches. In particular, in this case we do not need to analyze the points $P_{ \pm \infty}$, since they are either non-real, or real isolated points of $\mathcal{C}$, which we do not consider.

Finally, we compute the images $P_{i}=\mathbf{x}\left(Q_{i}\right)$, and we connect them according to how the $Q_{i}$ are connected in $\mathcal{G}$. The graph associated with $\mathcal{G}$ is shown in Fig. 4 (left). The graph associated with $\mathcal{C}$ is also shown in Fig. 4 (right). Additionally, in the graph associated there are several points very close to each other: some details on the topology of $\mathcal{C}$ are given in Fig. 5 .

\section{The space case.}

Here we consider $\mathbf{x}: \mathbb{R}^{2} \rightarrow \mathbb{R}^{3}$, where

$$
\mathbf{x}(t, s)=(x(t, s), y(t, s), z(t, s))=\left(\frac{A_{1}(t, s)}{B_{1}(t, s)}, \frac{A_{2}(t, s)}{B_{2}(t, s)}, \frac{A_{3}(t, s)}{B_{3}(t, s)}\right) .
$$

We let $\mathcal{C}=\mathbf{x}(\mathcal{G})$, where $\mathcal{G}$ is defined by Eq. (2). In this case, we follow the same stragegy already used in papers like [12, 14, 18]: first, birationally project $\mathcal{C}$ onto the $x y$-plane, then compute the topology of the projection (in our case, using the results in Section 3), and then lift this projection to get the topology of the curve $\mathcal{C}$.

Let $\mathcal{C}^{\star}=\pi_{x y}(\mathcal{C})$, where $\pi_{x y}$ denotes the projection onto the $x y$-plane, and let $\tilde{\mathbf{x}}=\pi_{x y} \circ \mathbf{x}$. Fig. 6 illustrates the relationship between $\mathcal{G}, \mathcal{C}$ and $\mathcal{C}^{\star}$. We need two hypotheses this time: 

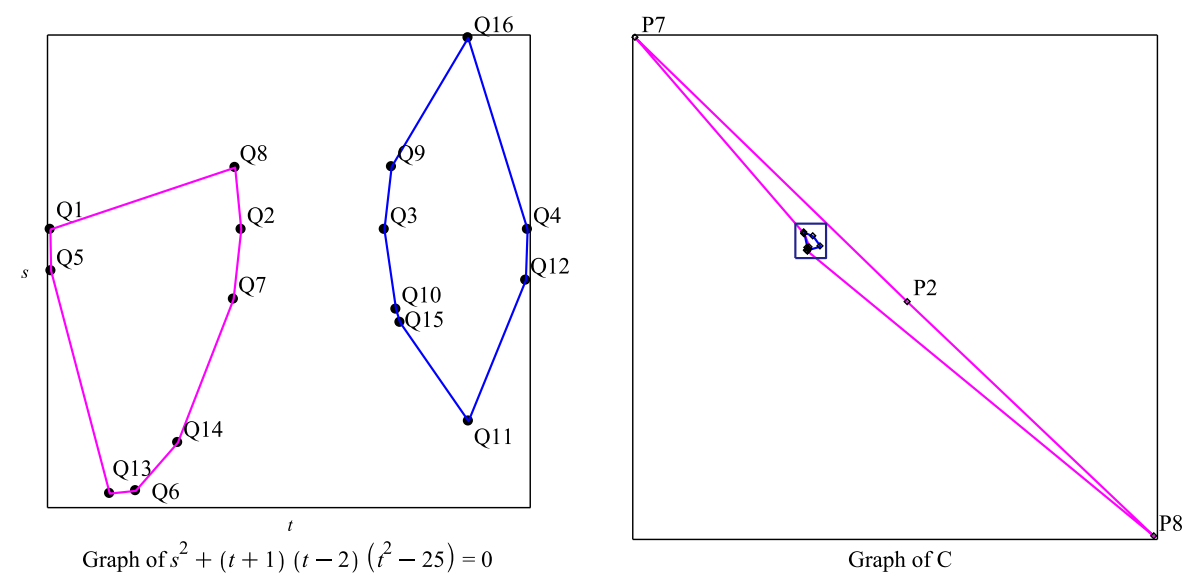

Figure 4: Correspondence between the edges of $G_{\mathcal{G}}$ and $G_{\mathcal{C}}$.

(H1) The restriction $\left.\tilde{\mathbf{x}}\right|_{\mathcal{G}}$ is birational.

(H2) The curve $\mathcal{C}^{\star}$ does not have any asymptotes parallel to either the $y$-axis, or the $z$-axis.

It is also customary, when computing the topology of a space curve $\mathcal{C}$, to require that $\mathcal{C}$ has no component parallel to the $z$-axis. However, in our case $\mathcal{C}$ is irreducible, i.e. $\mathcal{C}$ consists of only one component. If $\mathcal{C}$ reduces to a line parallel to the $z$-axis, then the only possibility is that both $x(t, s), y(t, s)$ are constant, which is a trivial case.

Hypothesis (H1) implies that $\mathbf{x}$ itself is birational when restricted to $\mathcal{G}$, and that $\pi_{x y}$ is also birational when restricted to $\mathcal{C}$; in turn, this means that there are not two different branches of $\mathcal{C}$ projecting as a same branch of $\mathcal{C}^{\star}$, and therefore that the branches of $\mathcal{C}$ are the result of lifting to space the branches of the projection $\mathcal{C}^{\star}=\pi_{x y}(\mathcal{C})$. Hypothesis (H1) can be checked, as observed in Section 3 , by taking a random point $\left(t_{0}, s_{0}\right) \in \mathcal{G}$ and determining the preimages of $\tilde{\mathbf{x}}\left(t_{0}, s_{0}\right)$. Hypothesis (H2) can be checked by testing whether or not $B_{2}(t, s)=g(t, s)=0$ has some solution where $A_{2}(t, s) \cdot B_{1}(t, s) \neq$ 0 , and whether or not $A_{2}(t, s)=g(t, s)=0$ has some solution where $A_{1}(t, s) \cdot B_{2}(t, s) \neq 0$. Both hypotheses, (H1) and (H2), guarantee that: (i) the topology of $\mathcal{C}^{\star}$ could be computed by applying the ideas in Section 3 ; (ii) the topology of $\mathcal{C}$ could be computed from the topology of $\mathcal{C}^{\star}$, by lifting 

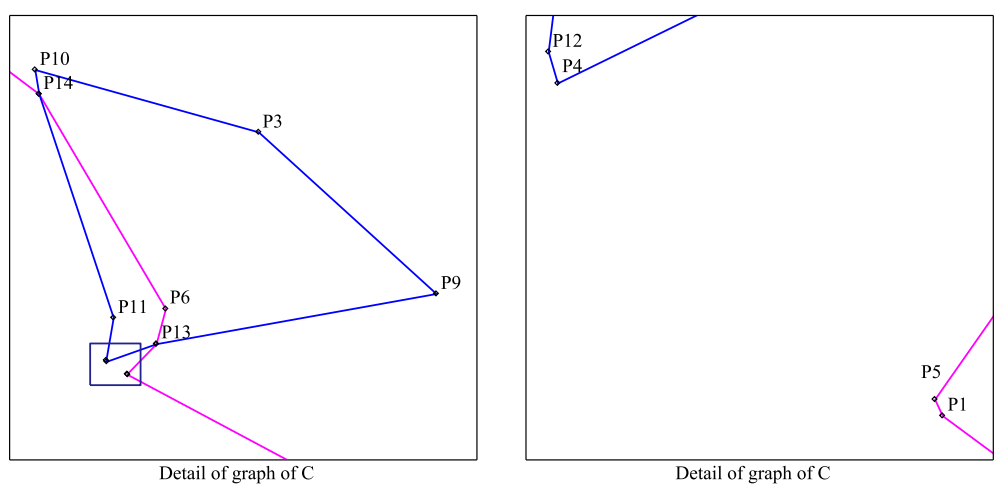

Figure 5: Details

a (planar) graph isotopic to $\mathcal{C}^{\star}$. In our case, however, we do not need to compute first the topology of $\mathcal{C}^{\star}$; instead, as in Section 3, we determine all the points $(t, s) \in \mathcal{G}$ giving rise to "notable" points of $\mathcal{C}$, and incorporate those points as vertices of $G_{\mathcal{G}}$. Then the edges of $G_{\mathcal{G}}$ are mapped onto edges of $G_{\mathcal{C}}$ as we did in Section 3 .

Hypotheses (H1) and (H2) can always be achieved when $\left.\mathbf{x}\right|_{\mathcal{G}}$ is birational. Indeed, under this assumption, for almost all random affine changes of coordinates $\phi$ and renaming $\mathbf{x}:=\mathbf{x} \circ \phi,\left.\pi_{x y}\right|_{\mathcal{C}}$ is birational, i.e. two different branches of $\mathcal{C}$ do not project as a same branch of $\mathcal{C}^{\star}$. As a consequence $\left.\tilde{\mathbf{x}}\right|_{\mathcal{G}}$ must be birational.

In this case, we need to include the following points as vertices of $G_{\mathcal{G}}$ :

(i) Critical points of $g(t, s)=0$, i.e. points of $\mathcal{G}$ where $g_{s}=0$.

(ii) Points of $\mathcal{G}$ giving rise to critical points of $\mathcal{C}^{\star}$.

(iii) Points of $\mathcal{G}$ where some component of $\mathbf{x}$ is not defined.

(iv) Starting and ending points for open branches of $\mathcal{G}$.

The points in (i), (ii), (iii) are computed as in Section 3; observe that the pairs $(t, s)$ generating singularities and points of $\mathcal{C}$ with tangent parallel to the $z$-axis are among the critical points of $\mathcal{C}^{\star}$ (see [5, 4]). Once the points $Q_{i}=\left(t_{i}, s_{i}\right), i=1, \ldots, r$ in (i)-(iv) are computed, we can find, whenever they are defined, the images $P_{i}=\mathbf{x}\left(Q_{i}\right)$ or the limit points and proceed as in Section 3 in order to connect the $Q_{i}$. 


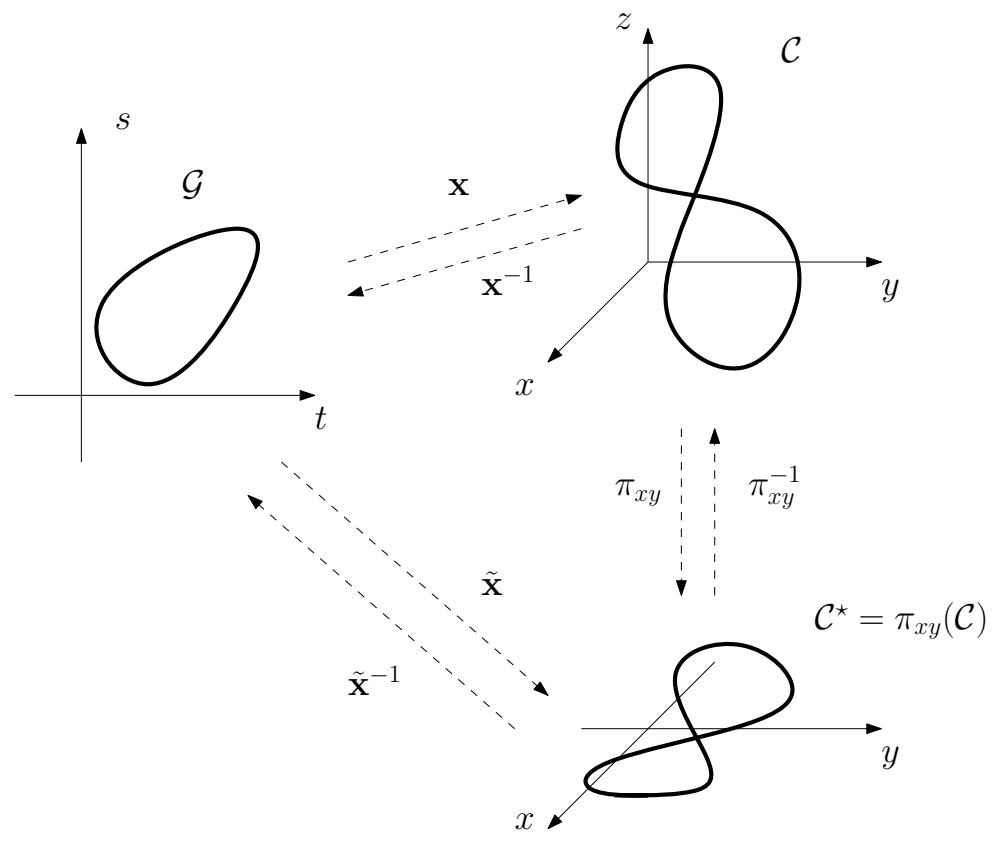

Figure 6: Relationship between the curves $\mathcal{G}, \mathcal{C}$, and $\mathcal{C}^{\star}$.

\section{Experimentation.}

In this section we report on the experimentation carried out in the case of both 2D and 3D curves. The algorithms have been implemented in Maple 2017, and the examples run on an Intel Core i3 processor with speeds revving up to $3.06 \mathrm{GHz}$.

Next, we first present examples of the $2 \mathrm{D}$ algorithm. In Table 1, we include for each curve, the genus, the total degree $\left(d_{i}\right)$ and the number of terms of the implicit equation (n.terms), the timings in seconds $\left(t_{0}\right)$ taken by our algorithm, and the timings in seconds $\left(t_{1}\right)$ corresponding to the algorithm in [21], also implemented in Maple, which uses the implicit equation of the curve. Additionally, in Table 1 we checkmark whether each example corresponds to a case where the points $P_{ \pm \infty}$ are affine (the column $P_{ \pm \infty}$ aff.), and whether the curve has self-intersections (S.I.). The last column provides some extra comments on the existence of base points or asymptotes. The parametrizations corresponding to these examples are given in Appendix II of [3, the ArXiv version of this paper. The graphs corresponding to the examples in Table 1 are shown in Figure (7); from left to right, we have 
Examples 1, 2, 3 in the first row, 4, 5, 6 in the second row and 7, 8, 9 in the

\begin{tabular}{|c|c|l|c|c|c|c|c|c|}
\hline Example & genus & $d_{i}$ & n.terms & $P_{ \pm \infty}$ aff. & S.I. & $t_{0}$ & $t_{1}$ & Obs. \\
\hline \hline 1 & 0 & 10 & 57 & $\checkmark$ & $\checkmark$ & 0.310 & 0.270 & Asymptotes \\
\hline 2 & 1 & 14 & 81 & & $\checkmark$ & 0.625 & ${ }^{*}$ & Asymptotes \\
\hline 3 & 2 & 6 & 26 & $\checkmark$ & $\checkmark$ & 0.398 & 0.110 & \\
\hline 4 & 1 & 12 & 81 & $\checkmark$ & $\checkmark$ & 0.529 & ${ }^{*}$ & Base points \\
\hline 5 & 2 & 12 & 75 & $\checkmark$ & $\checkmark$ & 0.543 & ${ }^{*}$ & \\
\hline 6 & 2 & 11 & 75 & & $\checkmark$ & 0.777 & ${ }^{*}$ & \\
\hline 7 & 2 & 12 & 75 & $\checkmark$ & $\checkmark$ & 0.443 & ${ }^{*}$ & \\
\hline 8 & 1 & 6 & 23 & $\checkmark$ & $\checkmark$ & 0.484 & 0.108 & \\
\hline 9 & 2 & 9 & 55 & $\checkmark$ & $\checkmark$ & 1.069 & 0.308 & \\
\hline
\end{tabular}

${ }_{622} *$ : Computation was cancelled after fifteen minutes.

${ }_{623}$ Notice that when the algorithm in [21] succeeds, it provides better timings ${ }_{624}$ than our algorithm. However, in most cases the implicit equation of the curve 625 is too big, and the algorithm in [21] gets stuck.

${ }_{626}$ Finally, we present examples of the 3D algorithm. In Table 2, for each ${ }_{627}$ curve we include the genus, the total degree $\left(d_{i}\right)$ and the number of terms ${ }_{628}$ of the implicit equation of the projection onto the $x y$-plane (n.terms), and ${ }_{629}$ the timing in seconds taken by our algorithm $\left(t_{0}\right)$; the parametrizations cor${ }_{630}$ responding to each curve are given in Appendix III of [3], the ArXiv version ${ }_{631}$ of this paper. Additionally, we include two columns on the nature of $P_{ \pm \infty}$ ${ }_{632}$ and the existence of self-intersections, as in Table 1. In the last column ${ }_{633}$ we include some observations on how we generated the example, in some 634 interesting cases. 


\begin{tabular}{|c|c|l|c|c|c|c|c|}
\hline Example & genus & $d_{i}$ & n.terms & $P_{ \pm \infty}$ aff. & S.I. & $t_{0}$ & Obs. \\
\hline \hline 1 & 4 & 10 & 66 & & & 1.543 & \\
\hline 2 & 2 & 6 & 16 & $\checkmark$ & & 0.344 & Int. con. and quadric \\
\hline 3 & 7 & 16 & 153 & & $\checkmark$ & 78.252 & Int. ruled and quadric \\
\hline 4 & 3 & 8 & 42 & & & 0.537 & Int. ruled and quadric \\
\hline 5 & 2 & 12 & 91 & & & 4.238 & Int. bicubic patch and plane \\
\hline 6 & 1 & 4 & 9 & & & 0.201 & \\
\hline 7 & 1 & 10 & 34 & & & 0.352 & \\
\hline 8 & 2 & 19 & 61 & $\checkmark$ & $\checkmark$ & 1.031 & \\
\hline 9 & 2 & 9 & 55 & $\checkmark$ & $\checkmark$ & 0.949 & \\
\hline
\end{tabular}

Table 2: 3D Examples.

The pictures corresponding to these curves are shown in Figure 8. Notice that the timing in Ex. 3 is considerably higher, which is expectable because both the Weierstrass curve and the mapping $\mathbf{x}(t, s)$ are dense and with high degree.

\section{Complexity and certification issues.}

In this section we present the complexity of the algorithms presented in the previous sections, and we elaborate on how to certificate the topology of the curves. To certify the topology we must be sure whether two different points $\left(t_{i}, s_{i}\right) \neq\left(t_{j}, s_{j}\right)$, both belonging to $\mathcal{G}$, satisfy $\mathbf{x}\left(t_{i}, s_{i}\right)=\mathbf{x}\left(t_{j}, s_{j}\right)$, that is whether they give rise to the same point $P \in \mathcal{C}$. We first analyze the complexity of the algorithm without the certification step: in particular, the timings corresponding to Section 5 do not include this certification. Then, we address certification issues and provide the complexity of the algorithm including the certification step. We analyze the algorithm for $3 \mathrm{D}$ curves: the complexity bound is the same for $2 \mathrm{D}$ and $3 \mathrm{D}$ curves.

\subsection{Complexity (I)}

In this section we present the bit complexity analysis of the algorithm without the certification step. This is the algorithm for which we perform experiments in Section 5. We denote the maximum bitsize by $\mathcal{L}(f)$ of the coefficients of a polynomial $f$. Additionally, we denote by $\mathcal{O}, \widetilde{\mathcal{O}}, \widetilde{\mathcal{O}}_{B}$ the arithmetic complexity, the arithmetic complexity neglecting logarithmic factors, and the bit complexity (also neglecting logarithmic factors), respectively. 
Let

$$
\mathbf{x}(t, s)=\left(\frac{a_{11}(t)+s a_{12}(t)}{b_{11}(t)+s b_{12}(t)}, \frac{a_{21}(t)+s a_{22}(t)}{b_{21}(t)+s b_{22}(t)}, \frac{a_{31}(t)+s a_{32}(t)}{b_{31}(t)+s b_{32}(t)}\right) .
$$

We consider the following 3 polynomials:

$$
\begin{aligned}
& X(t, s)=\left(b_{11}(t)+s b_{12}(t)\right) x-\left(a_{11}(t)+s a_{12}(t)\right) \\
& Y(t, s)=\left(b_{21}(t)+s b_{22}(t)\right) y-\left(a_{21}(t)+s a_{22}(t)\right), \\
& \left.Z(t, s)=\left(b_{31}(t)+s b_{32}(t)\right) z-\left(a_{31}(t)+s a_{32}(t)\right)\right) .
\end{aligned}
$$

We also recall that $g(t, s)=s^{2}-p(t)$. We assume that all the univariate polynomials in $t$, that is the $a_{i j}(t), b_{i j}(t)$, and $p(t)$, have degree at most $d$, and that their coefficients are integers of maximum bitsize at most $\tau$.

The process of the algorithm goes as follows: (Step 1) Compute the resultants

$$
E_{0}=\operatorname{res}_{s}(X, Y), E_{1}=\operatorname{res}_{s}(X, g) .
$$

The polynomial $E_{0}$ satisfies that $E_{0} \in \mathbb{Z}[x, y, t]$. The degree of $E_{0}$ with respect to $x$ and $y$ is 1 and with respect to $t$ is $\leq 2 d=\mathcal{O}(d)$; moreover $\mathcal{L}\left(E_{0}\right)=\widetilde{\mathcal{O}}(\tau)$. The polynomial $E_{1}$ satisfies that $E_{1} \in \mathbb{Z}[x, t]$. The degree of $E_{1}$ with respect to $x$ is 2 and with respect to $t$ is $\leq 3 d=\mathcal{O}(d)$; also $\mathcal{L}\left(E_{1}\right)=\widetilde{\mathcal{O}}(\tau)$.

Since the degree of $X, Y, Z$ and $g$ with respect to $x, y, s$ is at most 2, we can compute the resultants $E_{0}$ and $E_{1}$ by performing a constant number of multiplications of univariate polynomials in $t$. By recalling that the maximum degree with respect to $t$ is $\widetilde{\mathcal{O}}(d)$, we deduce that the cost of computing $E_{0}$ and $E_{1}$ is $\widetilde{\mathcal{O}}_{B}(d \tau)$ [30].

(Step 2) Compute the subresultant sequence of $E_{0}$ and $E_{1}$ with respect to $t$.

¿From the subresultant sequence we are interested in the polynomial of degree 1 with respect to $t$. This is the first subresultant polynomial; we can compute it in $\widetilde{\mathcal{O}}_{B}\left(d^{4} \tau\right)$ [16, Lemma 8]. Let the coefficient of degree 1 of this polynomial be $\operatorname{sres}_{1} \in \mathbb{Z}[x, y]$ (i.e. the first principal subresultant). It has degree $\widetilde{\mathcal{O}}(d)$ and bitsize $\widetilde{\mathcal{O}}(d \tau)$ [16, Lemma 8].

(Step 3) Substitute the parametrization $\mathbf{x}(t, s)$ in $\mathbf{s r e s}_{1}$.

After clearing denominators we obtain a polynomial $M(t, s) \in \mathbb{Z}[t, s]$. The degree of $M(t, s)$ with respect to $t$ and $s$ is $\widetilde{\mathcal{O}}(d)$ and its bitsize is $\widetilde{\mathcal{O}}\left(d^{2} \tau\right)$. This 
calculation of $M(t, s)$ involves $\mathcal{O}(d)$ multiplications of bivariate polynomials in $s$ and $t$. This cost is $\widetilde{\mathcal{O}}_{B}\left(d^{5} \tau\right)[25,30]$.

(Step 4) Solve the polynomial system $M(t, s)=g(t, s)=0$.

We can solve the system in $\widetilde{\mathcal{O}}_{B}\left(d^{7} \tau\right)\left(\right.$ or $\left.\widetilde{\mathcal{O}}_{B}\left(d^{8} \tau\right)\right)$ [19, 10].

After solving the system, we compute the images under the birational mapping $\mathbf{x}(t, s)$ of all the points $(t, s)$ computed along the way, and connect them properly.

The whole complexity is dominated by the complexity of solving the polynomial system $(\Sigma)\{M(t, s)=g(t, s)=0\}$, so we get a final bound of $\widetilde{\mathcal{O}}_{B}\left(d^{7} \tau\right)$ (or $\widetilde{\mathcal{O}}_{B}\left(d^{8} \tau\right)$ ), without including certification.

\subsection{Certification and complexity (II)}

In this subsection we consider certification strategies, and we present the complexity of the algorithm including this certification. We perform the certification by exploiting the rational univariate representation of the real roots of the polynomial system $(\Sigma)\{M(t, s)=g(t, s)=0\}$.

Within the complexity bound given in the previous subsection for solving the bivariate system $(\Sigma)$, we can compute both an isolating interval representation of the real roots, as a well a (sparse) rational univariate representation (SRUR) [10], see also [25]. The latter represents the tuples $(t, s)$ of the solutions os $(\Sigma)$ as $\left(\frac{F_{1}(\theta)}{F_{0}(\theta)}, \frac{F_{2}(\theta)}{F_{0}(\theta)}\right)$, where $\theta$ runs over all the (real) roots of a (univariate) polynomial $F(\theta)$ and $F_{0}, F_{1}$, and $F_{2}$ are univariate polynomials. This representation involves univariate polynomials of degree $\widetilde{\mathcal{O}}\left(d^{2}\right)$ and bitsize $\widetilde{\mathcal{O}}\left(d^{3} \tau\right)$.

Now we want to identify which tuples of solutions of the polynomial system $M(t, s)=g(t, s)=0$ give rise to the same point on space curve. Or in other words, we want to certify when two tuples give rise to the same point on the space curve.

Say that $\left(\alpha_{1}, \beta_{1}\right)$ and $\left(\alpha_{2}, \beta_{2}\right)$ are two different solutions of the polynomial system $(\Sigma)$. Assume further that they correspond to the roots $\theta_{1}$ and $\theta_{2}$ of the polynomial $F(\theta)$. Thus, their rational univariate representation is

$$
\left(\frac{F_{1}\left(\theta_{1}\right)}{F_{0}\left(\theta_{1}\right)}, \frac{F_{2}\left(\theta_{1}\right)}{F_{0}\left(\theta_{1}\right)}\right) \quad \text { and } \quad\left(\frac{F_{1}\left(\theta_{2}\right)}{F_{0}\left(\theta_{2}\right)}, \frac{F_{2}\left(\theta_{2}\right)}{F_{0}\left(\theta_{2}\right)}\right)
$$

with $F\left(\theta_{1}\right)=0, F\left(\theta_{2}\right)=0$. 
We check if they correspond to the same point by exploiting the parametrization $\mathbf{x}$. For example, to test if they result in the same $x$-coordinate, we should test whether or not

$$
\frac{a_{11}\left(\alpha_{1}\right)+\beta_{1} a_{12}\left(\alpha_{1}\right)}{b_{11}\left(\alpha_{1}\right)+\beta_{1} b_{12}\left(\alpha_{1}\right)}=\frac{a_{11}\left(\alpha_{2}\right)+\beta_{2} a_{12}\left(\alpha_{2}\right)}{b_{11}\left(\alpha_{2}\right)+\beta_{2} b_{12}\left(\alpha_{2}\right)} .
$$

Clearing denominators, we get $\widehat{G}\left(\alpha_{1}, \alpha_{2}\right)=0$. Now if we substitute the rational univariate representation of the roots and clear denominators, then we get a new bivariate polynomial $G$, and we need to test whether or not $G\left(\theta_{1}, \theta_{2}\right)=0$.

The degree of $G$ is $\widetilde{\mathcal{O}}\left(d^{3}\right)$, in $\theta_{1}$ and $\theta_{2}$ and its bitsize is $\widetilde{\mathcal{O}}\left(d^{4} \tau\right)$. The complexity of computing $G$ involves the multiplication of $\widetilde{\mathcal{O}}(d)$ univariate polynomials and is $\widetilde{\mathcal{O}}_{B}\left(d^{8} \tau\right)$. The cost of this bivariate sign evaluation is $\widetilde{\mathcal{O}}_{B}\left(d^{15} \tau\right)$.

We must perform this bivariate sign evaluation for every pair $\left(\theta_{i}, \theta_{j}\right)$ of roots of $F$, and test for all coordinates $(x, y, z)$. There are $\widetilde{\mathcal{O}}\left(d^{4}\right)$ pairs of solutions to test and the total cost is $\widetilde{\mathcal{O}}_{B}\left(d^{19} \tau\right)$. This complexity bound of certification dominates the overall complexity of the algorithm.

We have implemented the certification part and the timings we get are in agreement with this complexity: although there can be examples where the computing time is reasonable, in general the timings are very high and further research needs to be done. It seems plausible to improve the complexity of certification by exploiting more carefully aggregate separation bounds for the real roots of polynomial systems [20]. For example, we can apply this aggregation when we perform the time consuming sign evaluation of $G$ over all the roots of the polynomial $F$. There should be a gain of a factor $d^{2}$ with this approach.

However, the most promising direction is to use more advanced (probabilistic) tests for checking equality of real algebraic numbers [9]. The reader might notice that we do not really need the actual sign evaluation of $G$ at two real algebraic numbers. What we really need is to test whether or not the evaluation of $G\left(\theta_{1}, \theta_{2}\right)$ is zero or not.

\subsection{Comparison of complexities with implicit algorithms.}

A possibility to compute the topology of $\mathcal{C}$ is to compute first an implicit representation of the curve, and then to apply an algorithm to complete the topology of an implicit curve. In the planar case, the implicit representation 
requires just one bivariate polynomial $f(x, y)$, that can be computed using Gröbner bases. Denoting the degree of $f(x, y)$ by $n$, and denoting by $\tau_{f}$ the bitsize of the coefficients of $f$, the complexity of computing the topology of $f(x, y)=0$ is $\widetilde{\mathcal{O}}_{B}\left(n^{6}+n^{5} \tau_{f}\right)$. In our case $n=\widetilde{\mathcal{O}}(d)$ and $\tau_{f}=\widetilde{\mathcal{O}}(d \tau)$, so we reach a complexity of $\widetilde{\mathcal{O}}_{B}\left(d^{6} \tau\right)$, certainly better than the bound we give in Subsection 6.2.

In the space case, however, the situation is much more difficult. An implicit representation of $\mathcal{C}$ requires to compute a basis for the ideal of the curve, which might have more than two polynomials. Even if $\mathcal{C}$ is implicitly defined by only two polynomials $f_{i}(x, y, z)$, with $i=1,2$, the known complexities for implicit algorithms are worse than ours. In [15], one has the bound $\widetilde{\mathcal{O}}\left(n^{21} \tau_{f}\right)$, where $n, \tau_{f}$ are bounds for the degrees and bitsizes of the $f_{i}$, respectively. For the same case, in [12] one has the bound $\widetilde{\mathcal{O}}\left(n^{37} \tau_{f}\right)$.

\section{Conclusion.}

We have presented algorithms to compute the topology of $2 \mathrm{D}$ and $3 \mathrm{D}$ hyperelliptic curves that do not require to compute or make use of the implicit representation of the curve. The main idea is to see the hyperelliptic curve as the image of a planar curve, the Weierstrass form of the curve, under a birational mapping of the plane or the space. Seeing the curve this way, the algorithms determines how the topology of the Weierstrass form changes when the birational mapping is applied. While a not completely certified algorithm produces good and fast results, a completely certified algorithm is much slower, although it is competitive in the space case, in terms of complexity, with algorithms using an implicit representation of the curve. Some lines of improvement to speed up the certification are suggested in the paper. We plan to exploit these ideas in the future to get a faster, certified, algorithm.

\section{References}

\section{References}

[1] J. G. Alcázar and J.R. Sendra. Local shape of offsets to algebraic curves. Journal of Symbolic Computation, 42:338-351, 2007.

[2] Juan Gerardo Alcázar, Jorge Caravantes, and Gema M Díaz-Toca. A new method to compute the singularities of offsets to rational plane 
curves. Journal of Computational and Applied Mathematics, 290:385402, 2015.

[3] Juan Gerardo Alcázar, Jorge Carvantes, Gema María Díaz Toca, and Elias Tsigaridas. ArXiv 1812.11498, 2018.

[4] Juan Gerardo Alcázar and Gema María Díaz-Toca. Topology of 2d and 3d rational curves. Computer Aided Geometric Design, 27(7):483-502, 2010 .

[5] Juan Gerardo Alcázar and J Rafael Sendra. Computation of the topology of real algebraic space curves. Journal of Symbolic Computation, 39(6):719-744, 2005.

[6] Eric Berberich, Pavel Emeliyanenko, Alexander Kobel, and Michael Sagraloff. Arrangement computation for planar algebraic curves. In Proceedings of the 2011 International Workshop on Symbolic-Numeric Computation, pages 88-98. ACM, 2012.

[7] Eric Berberich, Pavel Emeliyanenko, Alexander Kobel, and Michael Sagraloff. Exact symbolic-numeric computation of planar algebraic curves. Theoretical Computer Science, 491:1-32, 2013.

[8] Michal Bizzarri, Miroslav Lávička, and Jan Vršek. Piecewise rational approximation of square-root parameterizable curves using the weierstrass form. Computer Aided Geometric Design, 56:52-66, 2017.

[9] Johannes Blomer. Computing sums of radicals in polynomial time. In Foundations of Computer Science, 1991. Proceedings., 32nd Annual Symposium on, pages 670-677. IEEE, 1991.

[10] Yacine Bouzidi, Sylvain Lazard, Guillaume Moroz, Marc Pouget, Fabrice Rouillier, and Michael Sagraloff. Solving bivariate systems using rational univariate representations. J. Complex., 37(C):34-75, December 2016.

[11] Jorge Caravantes, Gema M Díaz-Toca, Laureano González-Vega, and Ioana Necula. An algebraic framework for computing the topology of offsets to rational curves. Computer Aided Geometric Design, 52:28-47, 2017. 
[12] Jin-San Cheng, Kai Jina, and Daniel Lazard. Certified rational parametric approximation of real algebraic space curves with local generic position method. Journal of Symbolic Computation, 58:18-40, 2013.

[13] Jinsan Cheng, Sylvain Lazard, Luis Peñaranda, Marc Pouget, Fabrice Rouillier, and Elias Tsigaridas. On the topology of planar algebraic curves. In Proceedings of the twenty-fifth annual symposium on Computational geometry, pages 361-370. ACM, 2009.

[14] Diatta Niang Daouda, Bernard Mourrain, and Olivier Ruatta. On the computation of the topology of a non-reduced implicit space curve. In Proceedings of the twenty-first international symposium on Symbolic and algebraic computation, pages 47-54. ACM, 2008.

[15] Daouda Diatta. Calcul effectif de la topologie de courbes et surfaces algebriques reelles. Ph. D. Thesis, Universite de Limoges, 2009.

[16] Dimitrios I Diochnos, Ioannis Z Emiris, and Elias P Tsigaridas. On the asymptotic and practical complexity of solving bivariate systems over the reals. Journal of Symbolic Computation, 44(7):818-835, 2009.

[17] Arno Eigenwillig, Michael Kerber, and Nicola Wolpert. Fast and exact geometric analysis of real algebraic plane curves. In Proceedings of the 2007 international symposium on Symbolic and algebraic computation, pages 151-158. ACM, 2007.

[18] Mohammed El Kahoui. Topology of real algebraic space curves. Journal of Symbolic Computation, 43(4):235-258, 2008.

[19] Pavel Emeliyanenko and Michael Sagraloff. On the complexity of solving a bivariate polynomial system. In Proceedings of the 37th International Symposium on Symbolic and Algebraic Computation, pages 154161. ACM, 2012.

[20] Ioannis Z Emiris, Bernard Mourrain, and Elias P Tsigaridas. The dmm bound: Multivariate (aggregate) separation bounds. In Proceedings of the 2010 International Symposium on Symbolic and Algebraic Computation, pages 243-250. ACM, 2010. 
[21] Laureano González-Vega and Ioana Necula. Efficient topology determination of implicitly defined algebraic plane curves. Computer aided geometric design, 19(9):719-743, 2002.

[22] Morris Hirsch. Differential Topology. Springer-Verlag, 1976.

[23] Shafarevich I.R. Basic Algebraic Geometry 1 (Third edition). SpringerVerlag, 2013.

[24] Alexander Kobel and Michael Sagraloff. On the complexity of computing with planar algebraic curves. Journal of Complexity, 31(2):206-236, 2015 .

[25] Angelos Mantzaflaris, Éric Schost, and Elias Tsigaridas. Sparse rational univariate representation. In ISSAC 2017-International Symposium on Symbolic and Algebraic Computation, page 8, 2017.

[26] Alfred Menezes, Robert Zuccherato, and Yi-Hong Wu. An elementary introduction to hyperelliptic curves. Reseach Report CORR 96-19, Faculty of Mathematics, University of Waterloo, 1996.

[27] J Rafael Sendra, David Sevilla, and Carlos Villarino. Algebraic and algorithmic aspects of radical parametrizations. Computer Aided Geometric Design, 55:1-14, 2017.

[28] Juan Rafael Sendra, Franz Winkler, and Sonia Pérez-Díaz. Rational Algebraic Curves: A Computer Algebra Approach. Springer Verlag, 2007.

[29] G.M. Díaz Toca. http://webs.um.es/gemadiaz/miwiki/doku.php? id=papers, 2018.

[30] Joachim Von Zur Gathen and Jürgen Gerhard. Modern computer algebra. Cambridge university press, 2013.

[31] R.J. Walker. Algebraic Curves. Princeton University Press, 1950. 


\section{Appendix I: remaining proofs.}

In this section we provide the proofs of some results in Section 3 . We start with Proposition 5.

Proof. (of Proposition 5) Let $\mathcal{V}$ be the variety (the curve) in $\mathbb{R}^{4}(t, s, x, y)$ defined as

$$
\mathcal{V}=V(\operatorname{num}(x-x(t, s)), \operatorname{num}(y-y(t, s)), g(t, s)),
$$

and let $\widehat{\mathcal{V}}=\Pi_{t x y}(\mathcal{V})$ be the projection of $\mathcal{V}$ onto $\mathbb{R}^{3}(t, x, y)$; notice that $\widehat{\mathcal{V}} \subset V\left(\xi_{1}, \xi_{2}\right)$. Suppose that $\left(t_{0}, s_{0}, x_{0}, y_{0}\right)$ is smooth in $\mathcal{V}$. Using the Jacobian matrix of $F_{1}(t, s, x)=\operatorname{num}(x-x(t, s)), F_{2}(t, s, y)=\operatorname{num}(y-y(t, s)), g(t, s)$ and condition (11), we observe that the tangent line to $\mathcal{V}$ at $\left(s_{0}, t_{0}, x_{0}, y_{0}\right)$ is parallel to $\left(-g_{s}\left(t_{0}, s_{0}\right), g_{t}\left(t_{0}, s_{0}\right), 0,0\right)$. If $g_{s}\left(t_{0}, s_{0}\right) \neq 0$ (i.e. if $\left.s_{0} \neq 0\right)$ then the point $\left(t_{0}, x_{0}, y_{0}\right)$ is regular in $\widehat{\mathcal{V}}$ and the tangent line to $\widehat{\mathcal{V}}$ at $\left(t_{0}, x_{0}, y_{0}\right)$ is $\left\{x=x_{0}, y=y_{0}\right\}$, which is parallel to the $t$-axis. Therefore, $\xi_{1}\left(t, x_{0}\right)=0$ and $\xi_{2}\left(t, x_{0}, y_{0}\right)=0$ share the root $t_{0}$ with multiplicity higher than 1 , and $\operatorname{sres}_{1}\left(x_{0}, y_{0}\right)=0$. If $g_{s}\left(t_{0}, s_{0}\right)=0$ (i.e. if $\left.s_{0}=0\right)$ then $\left(t_{0}, x_{0}, y_{0}\right)$ is singular in $\widehat{\mathcal{V}}$ and we can derive the same conclusion.

If, however, $\left(s_{0}, t_{0}, x_{0}, y_{0}\right)$ is a singular point of $\widehat{\mathcal{V}}$, then the tangent space to $\mathcal{V}$ at $\left(s_{0}, t_{0}, x_{0}, y_{0}\right)$, i.e. the kernel of the Jacobian matrix, consists of the vectors $(\alpha, \beta, 0,0)$ with $\alpha, \beta \in \mathbb{C}$. Therefore, the line $\left\{x=x_{0}, y=y_{0}\right\}$ is tangent to $\widehat{\mathcal{V}}$ at $\left(t_{0}, x_{0}, y_{0}\right)$ and, therefore, all $\xi_{i}\left(t, x_{0}, y_{0}\right), i=1,2$ have a multiple root at $t=t_{0}$. This implies that $\operatorname{sres}_{1}\left(x_{0}, y_{0}\right)=0$.

Now we prove Lemma 7. From definitions of $\xi_{1}, \xi_{2}$ in Eq. (8) and taking into account that $\mathbf{x}$ can be written as in Eq. (4), the polynomial $\xi_{1}(t, x)$ is the square-free part of the resultant with respect to $s$ of $g(t, s)=s^{2}-p(t)$ and

$$
\begin{aligned}
h(t, s, x) & :=\operatorname{num}(x-x(t, s))= \\
& =x\left(b_{11}(t)+s b_{12}(t)\right)-\left(a_{11}(t)+s a_{12}(t)\right)= \\
& =s\left(x b_{12}(t)-a_{12}(t)\right)+x b_{11}(t)-a_{11}(t) .
\end{aligned}
$$

Since $\operatorname{degree}_{s}(g)=2$ and $\operatorname{degree}_{s}(h) \leq 1$, it is easy to compute such a resultant; if $\operatorname{degree}_{s}(h)=1$, i.e. if $x(t, s)$ explicitly depends on $s$, then

$$
\operatorname{Res}_{s}(h, g)=\left(b_{11}^{2}-p b_{12}^{2}\right) x^{2}-2\left(a_{11} b_{11}-p a_{12} b_{12}\right) x+a_{11}^{2}-p a_{12}^{2},
$$


where $b_{i j}=b_{i j}(t), a_{i j}=a_{i j}(t)$ for $i=1,2, j=1,2$. If $\operatorname{degree}_{s}(h)=0$, i.e. if $x(t, s)$ does not depend on $s$, then

$$
\operatorname{Res}_{s}(h, g)=h=x\left(b_{11}(t)+s b_{12}(t)\right)-\left(a_{11}(t)+s a_{12}(t)\right) .
$$

As for $\xi_{2}(t, x, y)$, that is is the square-free part of the resultant with respect to $s$ of $h(t, s, x)$ and

$$
\begin{aligned}
j(t, s, y) & :=\operatorname{num}(y-y(t, s))= \\
& =y\left(b_{21}(t)+s b_{22}(t)\right)-\left(a_{21}(t)+s a_{22}(t)\right)= \\
& =s\left(y b_{22}(t)-a_{22}(t)\right)+y b_{21}(t)-a_{21}(t) .
\end{aligned}
$$

If degree $_{s}(h)=\operatorname{degree}_{s}(j)=1$, i.e. if both $x(t, s)$ and $y(t, s)$ explicitly depend on $s$, then

$\operatorname{Res}_{s}(h, j)=\left(a_{22} b_{11}-a_{21} b_{12}\right) x+\left(a_{11} b_{22}-a_{12} b_{21}\right) y+\left(b_{12} b_{21}-b_{11} b_{22}\right) x y-a_{11} a_{22}+a_{12} a_{21}$,

where $b_{i j}=b_{i j}(t), a_{i j}=a_{i j}(t)$ for $i=1,2, j=1,2$. If degree $_{s}(h)=0$, i.e. if $x(t, s)$ does not depend on $s$, then

$$
\operatorname{Res}_{s}(h, j)=h=x\left(b_{11}(t)+s b_{12}(t)\right)-\left(a_{11}(t)+s a_{12}(t)\right),
$$

and if $\operatorname{degree}_{s}(j)=0$, i.e. if $y(t, s)$ does not depend on $s$, then

$$
\operatorname{Res}_{s}(h, j)=j=y b_{21}(t)-a_{21}(t) .
$$

Proof. (of Lemma 7) " $\Leftarrow$ " Suppose that $t_{0}$ corresponds to a base point. The resultant of $h(t, s, x)$ and $g(t, s)$ is equal to Equation (13), and considered as a polynomial in $x$, it is easy to see that all its coefficients vanish at $t=t_{0}$. Thus, $t-t_{0}$ divides $\xi_{1}(x, t)$. Likewise, the resultant of $h(t, s, x)$ and $j(t, s, y)$ is equal to Equation (15), and we can check that all its coefficients vanish in $t=t_{0}$. Thus, $t-t_{0}$ divides also $\xi_{2}(x, t)$.

" $\Rightarrow$ " If $t-t_{0}$ divides $\xi_{1}$, then, by properties of resultants, since the leading coefficient of $g(t, s)$ with respect to $s$ is 1 , there is $s_{0}$ with $g\left(t_{0}, s_{0}\right)=0$ and

$$
h\left(t_{0}, s_{0}, x\right)=x\left(b_{11}\left(t_{0}\right)+s_{0} b_{12}\left(t_{0}\right)\right)-\left(a_{11}\left(t_{0}\right)+s_{0} a_{12}\left(t_{0}\right)\right)=0 ;
$$

thus, $b_{11}\left(t_{0}\right)+s_{0} b_{12}\left(t_{0}\right)=a_{11}\left(t_{0}\right)+s_{0} a_{12}\left(t_{0}\right)=0$. 
Next, if $t-t_{0}$ divides $\xi_{2}$, then either the leading coefficients of both $h(t, s, x)$ and $j(t, s, y)$ with respect to $s$ vanish at $t=t_{0}$, or there exists $s_{1}$ such that $h\left(t_{0}, s_{1}, x\right)=j\left(t_{0}, s_{1}, y\right)=0$ for all $x, y$. In the first case, we would have

$$
b_{12}\left(t_{0}\right)=a_{12}\left(t_{0}\right)=b_{22}\left(t_{0}\right)=a_{22}\left(t_{0}\right)=0 .
$$

However, since also $b_{11}\left(t_{0}\right)+s_{0} b_{12}\left(t_{0}\right)=a_{11}\left(t_{0}\right)+s_{0} a_{12}\left(t_{0}\right)=0$, we should have

$$
a_{11}\left(t_{0}\right)=a_{12}\left(t_{0}\right)=b_{12}\left(t_{0}\right)=b_{11}\left(t_{0}\right)=0,
$$

but this cannot happen because $\operatorname{gcd}\left(a_{11}, a_{12}, b_{11}, b_{12}\right)=1$. Therefore, there exists $s_{1}$ such that for all $x, y$

$$
h\left(t_{0}, s_{1}, x\right)=x\left(b_{11}\left(t_{0}\right)+s_{1} b_{12}\left(t_{0}\right)\right)-\left(a_{11}\left(t_{0}\right)+s_{1} a_{12}\left(t_{0}\right)\right)=0 ;
$$

$$
j\left(t_{0}, s_{1}, y\right)=y\left(b_{21}\left(t_{0}\right)+s_{1} b_{22}\left(t_{0}\right)\right)-\left(a_{21}\left(t_{0}\right)+s_{1} a_{22}\left(t_{0}\right)\right)=0 .
$$

Then,

$$
\begin{aligned}
& b_{11}\left(t_{0}\right)+s_{1} b_{12}\left(t_{0}\right)=a_{11}\left(t_{0}\right)+s_{1} a_{12}\left(t_{0}\right)=0 \\
& b_{21}\left(t_{0}\right)+s_{1} b_{22}\left(t_{0}\right)=a_{21}\left(t_{0}\right)+s_{1} a_{22}\left(t_{0}\right)=0 .
\end{aligned}
$$

Since we also know that $b_{11}\left(t_{0}\right)+s_{0} b_{12}\left(t_{0}\right)=a_{11}\left(t_{0}\right)+s_{0} a_{12}\left(t_{0}\right)=0$, with $\left(t_{0}, s_{0}\right) \in \mathcal{G}$, we deduce that either $s_{1}=s_{0}$, or $b_{12}\left(t_{0}\right)=a_{12}\left(t_{0}\right)=0$. However, $b_{12}\left(t_{0}\right)=a_{12}\left(t_{0}\right)=0$ implies that $b_{11}\left(t_{0}\right)=a_{11}\left(t_{0}\right)=0$, which cannot happen because $\operatorname{gcd}\left(a_{11}, a_{12}, b_{11}, b_{12}\right)=1$. $s_{0}=s_{1}$ with $g\left(t_{0}, s_{0}\right)=0$. So, we can conclude that $t_{0}$ corresponds to a base point of $\mathbf{x}$.

Finally, we prove Lemma 8 .

Proof. (of Lemma 8) " $\Leftarrow$ " If $x(t, s)=x(t)$, then $\xi_{1}(t, x)=\xi_{2}(t, x, y)=$ $b_{11}(t) x-a_{11}(t)$, and the result follows.

" $\Rightarrow$ " By way of contradiction, suppose that $\xi_{1}(t, x)$ and $\xi_{2}(t, x, y)$ have a factor $\eta(t, x)$ depending on both $x, t$ and that $x(t, s)$ also depends on $s$. Notice that taking Eq. (17) into account, if $\xi_{1}(t, x)$ and $\xi_{2}(t, x, y)$ have a factor $\eta(t, x)$ depending on both $x, t$ then $y(t, s)$ must depend on $s$ as well. So both $x(t, s)$ and $y(t, s)$ depend on $s$. Then $\xi_{2}(t, x, y)$ is the square-free part of Eq. (15), so $\eta(t, x)$ must be linear in $x$. Therefore either $\xi_{2}(t, x, y)$ coincides with $\eta(t, x)$, or $\xi_{2}(t, x, y)$ has another factor $\gamma(t, y)$ whose degree in $y$ is at most 1 . Now we distinguish two cases: 
(i) If $\operatorname{degree}_{y}(\gamma)=1$, then for all $\left(t_{0}, y_{0}\right)$ such that $\gamma\left(t_{0}, y_{0}\right)=0$, either the leading coefficients of $h, j$ with respect to $s$ vanish at $\left(t_{0}, y_{0}\right)$ for all $x$, or there exists $s_{0}$ such that $h, j$ integrally vanish at $\left(t_{0}, s_{0}, y_{0}\right)$ for all $x$. The first possibility implies that both leading coefficients are zero modulo $\gamma(t, y)$, and this cannot happen because the leading coefficient of $h$ with respect to $s$ depends on $x$. But the second possibility cannot happen either, because that would imply that $x(t, s)$ has infinitely many base points.

(ii) If $\operatorname{degree}_{y}(\gamma)=0$, then for all $\left(t_{0}, x_{0}\right)$ such that $\eta\left(t_{0}, x_{0}\right)=0$, either the leading coefficients of $h, j$ with respect to $s$ vanish at $\left(t_{0}, x_{0}\right)$ for all $y$, or there exists $s_{0}$ such that $h, j$ integrally vanish at $\left(t_{0}, s_{0}, y_{0}\right)$ for all $y$. Then we argue as before, this time with $j$ and $y(t, s)$.

Thus we conclude that $x(t, s)$ cannot depend explicitly on $s$, and the result follows. 

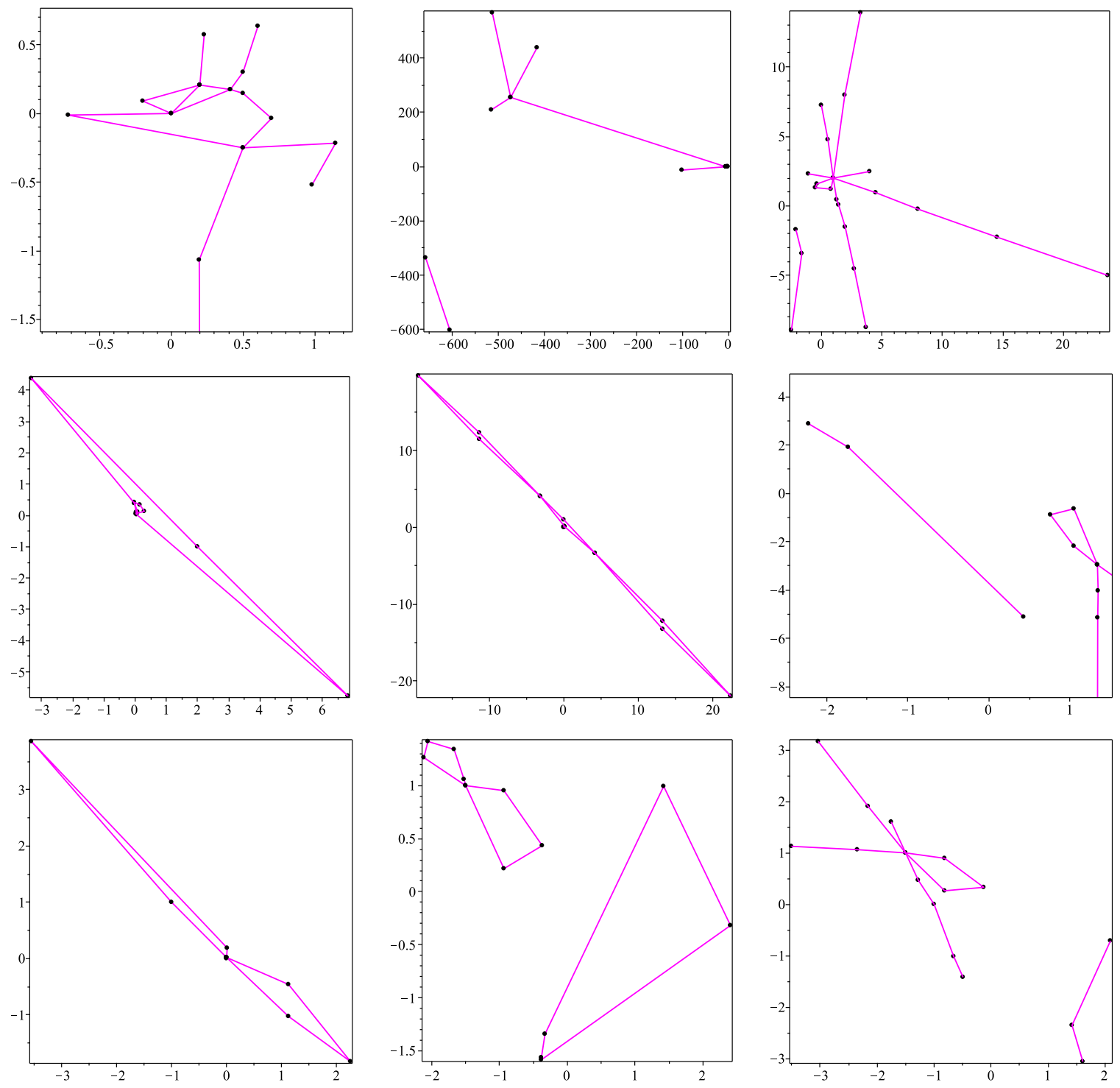

Figure 7: Examples of the 2D algorithm. 

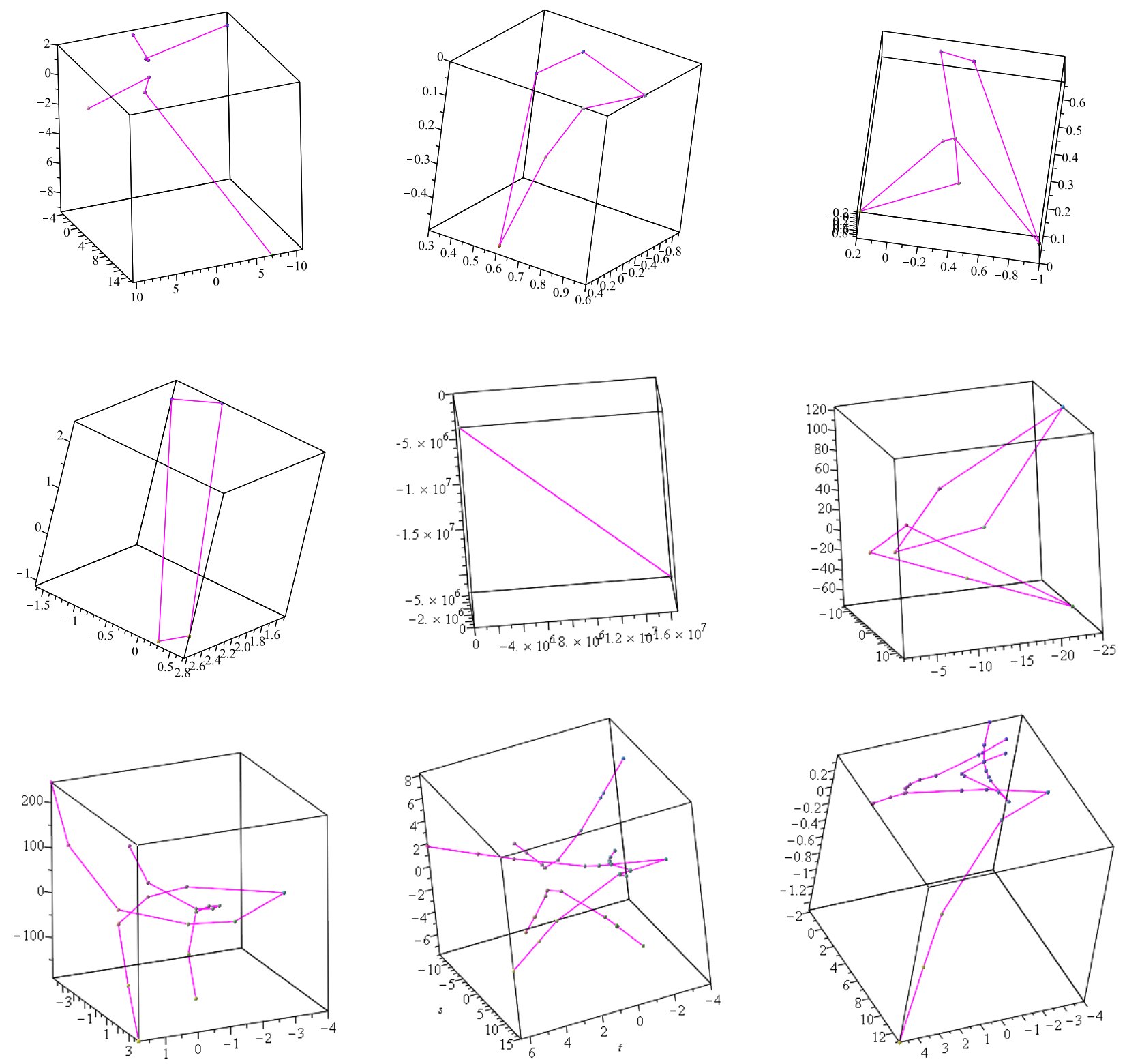

Figure 8: Examples of the 3D algorithm. 